\title{
Marine mammals and the community structure of the Estuary and Gulf of St Lawrence, Canada: evidence from stable isotope analysis
}

\author{
Véronique Lesage $^{1,2, *}$, Mike O. Hammill ${ }^{1}$, Kit M. Kovacs ${ }^{3,4}$ \\ ${ }^{1}$ Maurice Lamontagne Institute, Department of Fisheries and Oceans, PO Box 1000, Mont-Joli, Quebec G5H 3Z4, Canada \\ ${ }^{2}$ Department of Biology, University of Waterloo, Waterloo, Ontario N2L 3G1, Canada \\ ${ }^{3}$ Norwegian Polar Institute, 9296 Tromsø, Norway \\ ${ }^{4}$ Department of Biology, University of Waterloo, Waterloo, Ontario N2L 3G1, Canada
}

\begin{abstract}
The trophic relationships of both the benthic and pelagic communities in the Estuary and Gulf of St Lawrence regions were examined, with a special focus on the trophic position (TP) and relationship(s) among harbour, grey, hooded and harp seals and beluga whales. A multiple stable isotope and multiple tissue approach, used in conjunction with conventional dietary information, suggested that marine mammals occupied the highest trophic positions in the food webs of both communities and that they overlapped with one another to some extent trophically. Harbour seals Phoca vitulina and hooded seals Cystophora cristata occupied the highest TP, grey seals Halichoerus grypus, Gulf harp seals Phoca groenlandica, and male beluga whales Delphinapterus leucas were intermediate, and Estuary harp seals and female beluga whales were at the lowest TP. A general pattern of increasing enrichment of ${ }^{13} \mathrm{C}$ or ${ }^{15} \mathrm{~N}$ with age was observed in marine mammals (as well as fishes), although yearlings showed a decreased enrichment compared to both younger and older age classes. Sex also influenced $\delta^{15} \mathrm{~N}$ values. Males were more ${ }^{15} \mathrm{~N}$-enriched than females, with the difference between the sexes increasing with age, and being most pronounced in species that are sexually dimorphic with respect to body size. Geographical location also influenced isotope abundance. Estuary organisms were generally ${ }^{13} \mathrm{C}$-enriched relative to Gulf animals. $\delta^{13} \mathrm{C}$ values were on average lower in short-term diet integrators (blood serum) than in longer-term diet integrators (red blood cells) of harbour seals captured in April to June in the Estuary, which suggests that they probably did not move outside the Lower Estuary during the winter. Grey seals captured in the Lower Estuary did, however, show evidence of having been in the Gulf region some weeks or months before capture.
\end{abstract}

KEY WORDS: Stable isotopes $\cdot \delta^{13} \mathrm{C} \cdot \delta^{15} \mathrm{~N} \cdot$ Seal $\cdot$ Beluga whale $\cdot$ Food web $\cdot$ trophic position

\section{INTRODUCTION}

Marine mammals are generally top predators in marine ecosystems. However, little is known about their role in structuring marine ecosystems (Bowen 1997). This may be due in part to the fact that they tend to be large animals whose ecological needs often exceed the temporal and spatial scales used to define

\footnotetext{
*Present address: Maurice Lamontagne Institute.

E-mail: lesagev@dfo-mpo.gc.ca
}

community boundaries. Information on diet composition and trophic relationships of community components are needed to help understand the role of marine mammals in ecosystem dynamics.

Insights into trophic relationships and feeding habits of marine mammals may be gained by using ratios of naturally occurring isotopes, often isotopes of carbon and nitrogen, in specific tissues (e.g. Hobson \& Welch 1992, Ostrom et al. 1993, Muir et al. 1995). This method is based on the demonstration that stable isotope ratios in tissues of animals are correlated with those of their 
diet (Gearing et al. 1984, Minagawa \& Wada 1984, Hobson et al. 1996). When nitrogen ( $\mathrm{N}$ ) is assimilated, ${ }^{14} \mathrm{~N}$ is preferentially excreted and consumers become enriched in ${ }^{15} \mathrm{~N}$ relative to their food (Minagawa \& Wada 1984). Enrichment in ${ }^{15} \mathrm{~N}$ from 1 trophic level to the next is relatively predictable. This allows $\mathrm{N}$ isotopes to serve as indicators of a consumer's trophic position relative to that of species occupying lower trophic positions, e.g. primary producers. Carbon $\left({ }^{13} \mathrm{C}\right)$ is less useful as an index of trophic position because the trophic fractionation of ${ }^{13} \mathrm{C}$ is typically small compared to that of ${ }^{15} \mathrm{~N}$, resulting in relatively small enrichment in the heavier isotope $\left({ }^{13} \mathrm{C}\right)$ along the food chain (reviewed in Lesage 1999). Although this makes $\mathrm{C}$ a less reliable index of trophic position, it is still useful to the study of diet through its use as a tracer of sources of primary productivity. Primary producers vary in their isotopic $C$ signatures according to their origin (terrestrial or aquatic), $\mathrm{C}$ pool used and concentration $\left(\mathrm{CO}_{2}\right.$ in air, dissolved $\mathrm{CO}_{2}$ or $\mathrm{HCO}_{3}{ }^{-}$in water), prevailing $\mathrm{C}$ pathways $\left(\mathrm{C}_{3}\right.$ vs $\mathrm{C}_{4}$ plants), and the condition of the surrounding environment (well mixed or stagnant) (Smith \& Epstein 1971, Osmond et al. 1981, France 1995, Fry 1996). These properties assist in discriminating between animals which obtain their resources from terrestrial versus aquatic, benthic versus pelagic or inshore versus offshore environments (Ramsay \& Hobson 1991, Smith et al. 1996, Page 1997).

The stable isotope approach also provides diet estimates that are not affected by the differential rate of disappearance of prey in stomach contents or faeces. It bypasses the problems associated with the high frequency of empty stomachs found in marine mammals and estimates the assimilated, not just ingested, food. Furthermore, because the turnover time of isotopes is a function of a tissue's metabolic rate, the analysis of tissues with variable metabolic rates can provide information on diet integrated over a few days (e.g. blood plasma, liver) or a few months (e.g. red blood cells or muscle) (Tieszen et al. 1983, Hobson \& Clark 1992a, Hilderbrand et al. 1996). This diet assessment method is relatively non-invasive. It adds some flexibility to a sampling regime, in terms of both frequency and timing, since information on an animal's diet while in a particular region can be obtained a few weeks after its departure by sampling a long-term diet integrator such as red blood cells.

One drawback to the stable isotope approach is that it cannot identify which species from a given trophic level were consumed. Creating models that estimate the relative contribution of different prey to the diet of a consumer requires some basic knowledge of potential prey species. Owing to the rapidly increasing complexity of the calculations, these models generally assume that no more than 2 or 3 prey species are im- portant (Gearing 1991, Ben-David et al. 1997). The stable isotope approach also requires determination of the enrichment in ${ }^{13} \mathrm{C}$ and ${ }^{15} \mathrm{~N}$ at each trophic level (trophic enrichment factor, TEF). TEFs may vary depending on the tissue used, but appear relatively constant for the same tissue among species. TEFs have been determined for muscle tissue and for whole individuals of a variety of species fed a known diet (reviewed in Lesage 1999). In these experiments, TEFs for ${ }^{15} \mathrm{~N}$ usually ranged from 2.9 to $3.8 \%$, whereas TEFs for ${ }^{13} \mathrm{C}$ were more variable between studies, owing possibly in part to the failure to extract the ${ }^{13} \mathrm{C}$-depleted lipids from samples (reviewed in Lesage 1999).

In this study, we examine the trophic structure of the St Lawrence Estuary and Gulf communities and explore the relationships among 4 pinniped species (harbour seals Phoca vitulina, grey seals Halichoerus grypus, hooded seals Cystophora cristata, and harp seals Phoca groenlandica) and the beluga whale Delphinapterus leucas, using a multiple stable isotope $(\mathrm{C}$ and $\mathrm{N})$ and multiple tissue (blood red cells and serum, and hair) approach.

\section{MATERIALS AND METHODS}

Study area-Estuary and Gulf of St Lawrence. The St Lawrence River system is a highly productive region located in a temperate-subarctic climate in eastern Canada (Fig. 1; El-Sabh \& Silverberg 1990, Therriault 1991). The Estuary is naturally divided into 2 regions, hereafter called the Upper and Lower Estuary. Terrestrially-derived $\mathrm{C}$, freshwater phytoplankton and saltmarsh plants might be important $C$ sources for a few organisms from the Upper Estuary, since sediments of terrestrial origin, as well as salt-marsh and riverborne planktonic diatoms are found in this region (Tan \& Strain 1983). However, as one moves into the Lower Estuary and Gulf, salt marshes become less important, salinity increases and sediments become dominated by marine sources. The Gulf of St Lawrence, which begins near Pointe-des-Monts, is characterised by high salinities (>28\%) and unusually high levels of biological production relative to open seas. It is considered to be an inland sea because its internal current circulation limits exchange with the western North Atlantic (Therriault 1991).

Field collections. Marine mammals: Carbon and nitrogen isotopic signatures were obtained from 285 marine mammals: 112 harbour seals, 35 grey seals, 44 hooded seals, 57 harp seals, and 37 beluga whales (Table 1). Harbour and grey seals were live-captured in the Estuary by deploying a gill net near their haulout sites. Most were captured in the Lower Estuary at Bic and Pointe Mitis during May to October 1995 to 1997 (Fig. 1). A few individuals were captured at 
Table 1. Tissues collected from males $(\mathrm{M})$ and females $(\mathrm{F})$ of different marine mammals for the determination of isotope ratios of stable carbon and nitrogen. Harbour seal = Phoca vitulina; grey seal = Halichoerus grypus; hooded seal = Cystophora cristata; harp seal $=P$. groenlandica $;$ beluga $=$ Delphinapterus leucas

\begin{tabular}{|c|c|c|c|c|c|c|c|c|c|c|c|c|c|}
\hline \multirow{3}{*}{$\begin{array}{l}\text { Species } \\
\text { Region } \\
\text { (period) }\end{array}$} & \multirow{3}{*}{ Age class } & \multirow[t]{3}{*}{ Year } & \multirow{3}{*}{$\begin{array}{l}\text { Mean mass } \\
\pm \mathrm{SD}(\mathrm{kg})\end{array}$} & \multirow{3}{*}{$\begin{array}{c}\text { Mean age } \\
\pm \mathrm{SD}(\mathrm{yr})\end{array}$} & \multirow{3}{*}{ Total } & \multicolumn{8}{|c|}{ Number of samples taken } \\
\hline & & & & & & \multicolumn{2}{|c|}{ Blood cells } & \multicolumn{2}{|c|}{ Blood serum } & \multicolumn{2}{|c|}{ Muscle } & \multicolumn{2}{|c|}{ Hair } \\
\hline & & & & & & $\mathrm{F}$ & $\mathrm{M}$ & $\mathrm{F}$ & $\mathrm{M}$ & $\mathrm{F}$ & M & $\mathrm{F}$ & $\mathrm{M}$ \\
\hline \multicolumn{14}{|l|}{ Harbour seal } \\
\hline Lower Estuary & Nursing pup & 1995 & $19.4 \pm 5.9$ & 0 & 21 & 9 & 12 & 8 & 12 & - & - & - & - \\
\hline (Apr-Nov) & Weaned pup & 1995 & $23.9 \pm 4.6$ & 0 & 8 & 5 & 3 & 5 & 3 & - & - & - & - \\
\hline & & 1996 & $22.2 \pm 1.1$ & 0 & 6 & 4 & 2 & 4 & 2 & - & - & _- & _- \\
\hline & Yearling & 1995 & $36.2 \pm 5.4$ & 1 & 10 & 6 & 4 & 6 & 4 & - & - & 4 & 4 \\
\hline & & 1996 & $36.3 \pm 4.1$ & 1 & 13 & 3 & 10 & 2 & 9 & - & - & - & - \\
\hline & & 1997 & $35.8 \pm 2.6$ & 1 & 10 & 7 & 3 & - & - & - & - & - & - \\
\hline & $2+$ individuals & 1995 & $66.8 \pm 15.8$ & $5.5 \pm 2.0$ & 18 & 6 & 12 & 6 & 12 & - & - & 7 & 11 \\
\hline & & 1996 & $73.4 \pm 18.7$ & $6.9 \pm 3.2$ & 21 & 11 & 10 & 11 & 9 & - & - & - & - \\
\hline & & 1997 & $61.4 \pm 20.6$ & $3.6 \pm 2.2$ & 5 & 2 & 3 & - & - & - & - & - & - \\
\hline \multicolumn{14}{|l|}{ Beluga } \\
\hline Estuary & $2+$ individuals & 1988 & - & $21.9 \pm 3.8$ & 9 & - & - & - & - & 6 & 3 & - & - \\
\hline (Apr-Nov) & & 1989 & - & $22.1 \pm 5.2$ & 10 & _- & - & _- & - & 6 & 4 & _ & _- \\
\hline & & 1990 & - & $19.3 \pm 4.3$ & 8 & - & - & - & - & 4 & 4 & - & - \\
\hline & & 1997 & - & $21.9 \pm 8.9$ & 10 & - & - & - & - & 6 & 4 & - & - \\
\hline \multicolumn{14}{|l|}{ Grey seal } \\
\hline Lower Estuary & Yearling & 1995 & $53.6 \pm 16.2$ & 1 & 4 & 3 & 1 & 3 & 1 & - & - & - & _- \\
\hline (Apr-Nov) & & 1996 & $57.3 \pm 14.4$ & 1 & 6 & 5 & 1 & 5 & 1 & - & _- & _- & _- \\
\hline & $2+$ individuals & 1995 & $140.8 \pm 48.5$ & - & 3 & 1 & 2 & 1 & 2 & - & - & - & - \\
\hline & & 1996 & $95.5 \pm 13.5$ & - & 6 & 3 & 3 & 3 & 3 & - & - & - & - \\
\hline \multicolumn{14}{|l|}{ Port-Hood } \\
\hline $\begin{array}{l}\text { Nova Scotia } \\
\text { (Jan) }\end{array}$ & $2+$ individuals & 1996 & $200.1 \pm 47.6$ & $15.5 \pm 6.8$ & 16 & 8 & 8 & - & - & - & - & - & - \\
\hline \multicolumn{14}{|c|}{ Hooded seal } \\
\hline Magdalen Islands & $2+$ individuals & 1995 & $223.0 \pm 49.8$ & $17.2 \pm 6.8$ & 27 & 20 & 7 & - & - & - & - & - & - \\
\hline (Mar) & & 1996 & $252.9 \pm 37.9$ & $11.6 \pm 2.9$ & 17 & 8 & 9 & - & - & - & - & - & - \\
\hline \multicolumn{14}{|l|}{ Harp seal } \\
\hline Lower Estuary & Yearling & 1995 & $33.5 \pm 5.7$ & 1 & 9 & - & - & - & - & 4 & 5 & - & - \\
\hline (Feb) & $2+$ individuals & 1995 & $68.1 \pm 12.3$ & $3.9 \pm 0.8$ & 9 & - & - & _- & - & 7 & 2 & _- & _- \\
\hline Magdalen Islands & $2+$ individuals & 1995 & $110.4 \pm 23.4$ & $9.0 \pm 3.9$ & 24 & 18 & 6 & - & - & - & - & - & - \\
\hline (Mar) & & 1996 & $129.4 \pm 16.4$ & $8.9 \pm 3.9$ & 15 & 8 & 7 & - & - & - & - & - & - \\
\hline
\end{tabular}

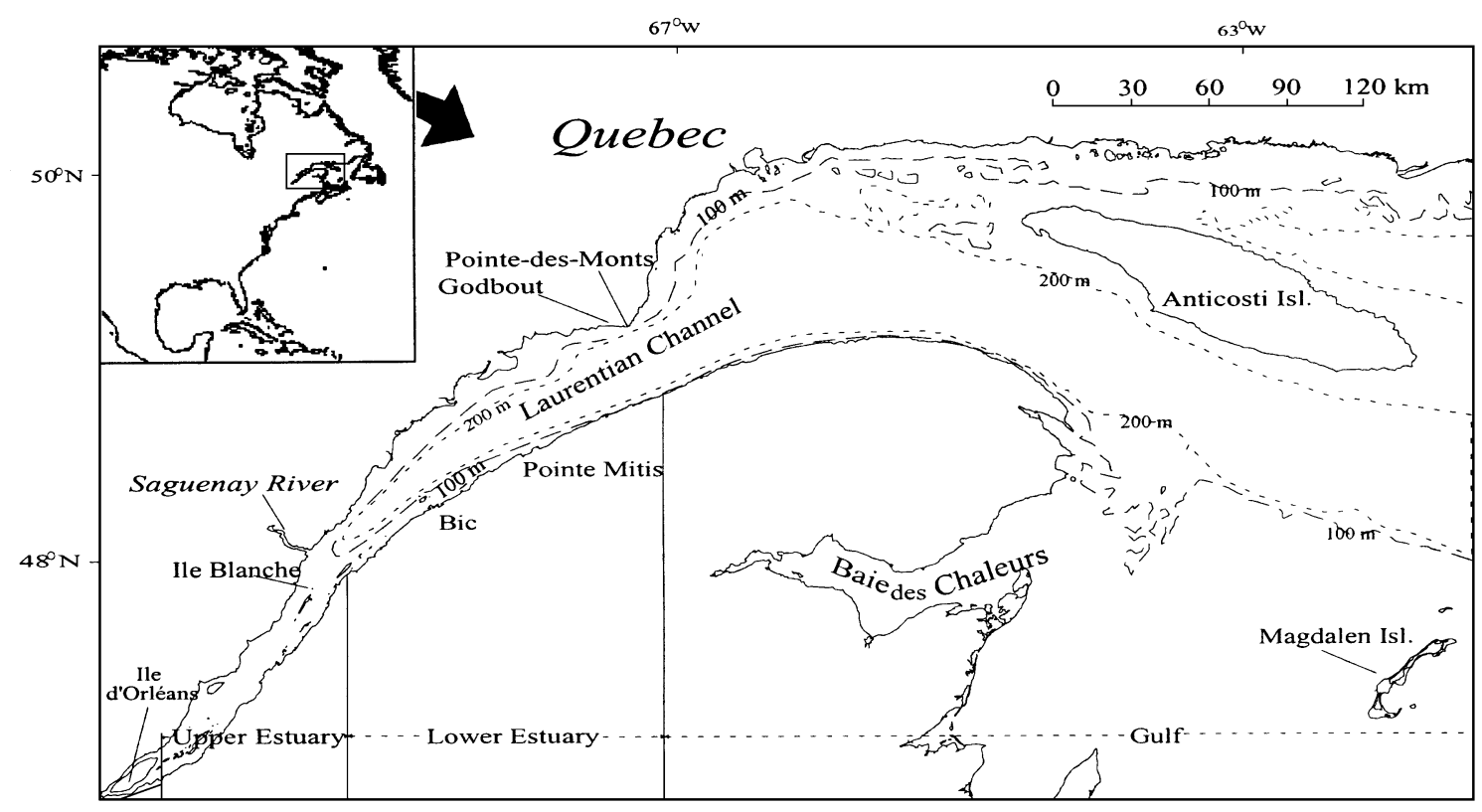

Fig. 1. Sampling area in Estuary and Gulf of St Lawrence, eastern Canada 
Ile Blanche, an island located at the limit of the Upper and Lower Estuary. Some grey seals (January) and harp seals (March), and all hooded seals (March) were captured live on the ice in the southern Gulf during their respective breeding periods. Some of these animals actually may have spent some time in the Estuary prior to their capture. Harp seals were also collected as part of the annual hunt in the Lower Estuary near Godbout in February. Beluga samples were obtained from fresh-beached carcasses in either the Estuary or Gulf of St Lawrence. Their stable isotope values were assumed not to have changed after death.

Live-captured seals were immobilised with an intramuscular injection of Telazol ${ }^{\circledR}$ (Tiletamine and Zolazepam, Fort Dodge ${ }^{\circledR}$ Laboratories, Iowa) at a dose of 0.5 to $1.0 \mathrm{mg} \mathrm{kg}^{-1}$ body mass, and weighed using one or two $200 \pm 0.5 \mathrm{~kg}$ Salter spring scales suspended from a portable tripod. A lower incisor was extracted for age determination. A blood sample was taken from the extradural vein and hair was clipped from the middorsal region. Animals sampled after death were also weighed and a sample of muscle tissue and a tooth (canine for seals, any tooth for beluga whales) were collected. Blood serum and clotted cells were separated by centrifugation. All samples were frozen separately at $-20^{\circ} \mathrm{C}$ until analyses.

Other components of Estuary and Gulf communities: Samples from species other than marine mammals ( $n=533$ ) were obtained from both the Estuary and Gulf of St Lawrence as part of ongoing research programs within the Department of Fisheries and Oceans. POM was collected using $4 \mathrm{l}$ Niskin bottles at the depth of maximum chlorophyll production and obtained by filtering water through pre-combusted Whatman GF/C filters. Zooplankton was collected concomitantly from vertical tows using a $1 \mathrm{~m}$ diam. $\times 3 \mathrm{~m}$ long Bongo net with $333 \mu \mathrm{m}$ mesh. Zooplankton were kept alive overnight to allow clearance of gut contents and were separated according to size and species. Zooplankton, larger invertebrates and fishes were stored at $-20^{\circ} \mathrm{C}$ until analyses. Depending on size, either a muscle sample (fishes and larger invertebrates including decapods) or the entire organism (other crustaceans) were used for analyses.

Chemical analyses. Prey samples and marine mammal blood and muscle tissues were freeze-dried for 36 to $48 \mathrm{~h}$ and homogenised. Hair was cleaned, using a detergent and an ultrasonic bath, rinsed several times in distilled water, and then oven-dried at $60^{\circ} \mathrm{C}$. The hair was then soaked in ether, rinsed in distilled water and allowed to air dry. The smaller plankton (e.g. copepods) were not acid-washed because carbonates have no observable effect on their $\delta^{13} \mathrm{C}$ values and this procedure may alter $\delta^{15} \mathrm{~N}$ values (Gearing et al. 1984, Pinnegar \& Polunin 1999). However, carbonates may inflate $\delta^{13} \mathrm{C}$ values by about $0.4 \%$ in slightly larger organisms such as krill (Bunn et al. 1995). Therefore, a correction factor was used for the $\mathrm{C}$ signatures of krill and species of comparable size such as Caprella sp. and Gammarus sp.

Lipids were extracted from all samples other than marine mammal tissues (Bligh \& Dyer 1959) since they are lighter in $\delta^{13} \mathrm{C}$ by $\sim 6 \%$ relative to protein (MCConnaughey \& McRoy 1979) and may add undesirable variability to $\delta^{13} \mathrm{C}$ values. The lipid content of marine mammal tissue samples can be predicted accurately from C:N ratios (Lesage 1999). Assuming that lipids and proteins are the 2 major constituents of blood red cells and serum and muscle, fat-free $\delta^{13} \mathrm{C}$ values were estimated by adding $6 \% \times \%$ fat to the measured $\delta^{13} \mathrm{C}$ values (Alexander et al. 1996).

Fur was analysed only for the 1995 harbour seal samples, and serum was analysed only for Estuary harbour and grey seals due to financial limitations (Table 1). Red blood cells and muscle were the tissues chosen for comparative analyses among the different species because these tissues integrated diet over relatively long periods ( $\geq 2$ to 3 mo: Tieszen et al. 1983, Hobson \& Clark 1992b, Hilderbrand et al. 1996).

Samples were analysed using an isochrom continuous-flow stable isotope mass spectrometer coupled to a Carla Erba elemental analyzer (CHNS-O EA1108) (Environmental Isotope Laboratory, University of Waterloo, Waterloo, Ontario). By convention, ${ }^{13} \mathrm{C}$ and ${ }^{15} \mathrm{~N}$ isotope abundance are expressed in delta notation (\%), relatively to the PeeDee Belemnite (PDB) and atmospheric nitrogen (AIR) standards, respectively:

$$
\delta X=\left[\left(R_{\text {sample }} / R_{\text {standard }}\right)-1\right] \times 1000
$$

where $X$ is ${ }^{13} \mathrm{C}$ or ${ }^{15} \mathrm{~N}$, and $R_{\text {sample }}$ is the corresponding ratio ${ }^{13} \mathrm{C} /{ }^{12} \mathrm{C}$ or ${ }^{15} \mathrm{~N} /{ }^{14} \mathrm{~N}$. $R_{\text {standard }}$ represents the ratios for their respective standards. Replicates using laboratory standards indicated an analytical error of \pm 0.1 and $\pm 0.3 \%$ for $\delta^{13} \mathrm{C}$ and $\delta^{15} \mathrm{~N}$, respectively. Deviations observed between replicates using samples in this study were on average $0.2 \%$ for both $\delta^{13} \mathrm{C}$ and $\delta^{15} \mathrm{~N}(\mathrm{n}=168)$.

Subdivision of marine mammal and fish species into classes. Ages of the marine mammals were determined to the nearest year by counting growth-layer groups of sectioned teeth (Brodie et al. 1990, Bernt et al. 1996). Harbour seal pups were defined as nursing pups if they were captured before July 10, a date corresponding to the date of first births and peak of pupping (May 25 to June 1) + mean duration of lactation (24 d) + $\sim 2$ wk post-lactation fast (Muelbert \& Bowen 1993). Weaned pups were defined as pups captured after July 10 until they were $\sim 5$ mo old.

When $\geq 5$ specimens of a given species of fish or large invertebrate were collected, $\delta^{13} \mathrm{C}$ and $\delta^{15} \mathrm{~N}$ values were 
linearly regressed against length to determine whether size affected $\mathrm{C}$ or $\mathrm{N}$ isotope ratios. Each species for which a significant $(\mathrm{p}<0.05)$ effect of size on either $\delta^{13} \mathrm{C}$ or $\delta^{15} \mathrm{~N}$ values was detected were segregated into length classes to increase group homogeneity of $\delta^{13} \mathrm{C}$ or $\delta^{15} \mathrm{~N}$ values (usually to $\mathrm{SD}<0.5 \%$ ). The withingroup standard deviation (WGSD) was used as a segregation criterion. The limit for a length class was defined as the length at which WGSD was minimal. To identify these limits, observations for a given species were sorted in ascending order of length. At first, only the smallest individual was included in Group 1, other observations being in Group 2. WGSD was calculated for each group when group size was $>1$. The second smallest animal was then added to Group 1, WGSD was re-calculated, and so on until all observations were contained in Group 1. When the limit between the 2 length classes was identified, observations with lengths above this limit were re-sorted in ascending order and the process was repeated until no further segregation was possible (i.e. until WGSD remained similar to total $\mathrm{SD}$ ). In most instances when size affected both $\delta^{13} \mathrm{C}$ and $\delta^{15} \mathrm{~N}$ for a given species, length classes identified using $\mathrm{C}$ or $\mathrm{N}$ isotopes corresponded. However, an adjustment of length classes was required in a few instances. The consequences of choosing length classes based on one isotope or the other on WGSD was examined; the combination which resulted in the smallest increase in WGSD was retained.

Isotopic model. To examine the trophic structure of the Estuary and Gulf communities, $\delta^{15} \mathrm{~N}$ signatures were converted to trophic position, TP, using:

$$
\mathrm{TP}=1+\left(D_{\mathrm{m}}-\mathrm{POM}\right) / \mathrm{TEF}
$$

where $D_{\mathrm{m}}=\delta^{15} \mathrm{~N}$ value in a consumers tissue, $\mathrm{POM}=$ $\delta^{15} \mathrm{~N}$ value of particulate organic matter, and TEF $=$ trophic enrichment factor in ${ }^{15} \mathrm{~N}$ for a particular tissue (Hobson \& Welch 1992). This latter value was set to $3.4 \%$ for all community components except marine mammals, which represents the average TEF obtained for muscle tissue or whole animals of a variety of freshwater or marine species other than marine mammals (reviewed in Lesage 1999). For the latter group of species, $\delta^{13} \mathrm{C}$ and $\delta^{15} \mathrm{~N}$ TEFs $\left(\mathrm{TEF}_{\mathrm{mmt}}\right)$ that were obtained from captive seals fed a known diet were used for blood red cells and serum, muscle and hair (Table 2). The TP of marine mammals was calculated using:

$$
\mathrm{TP}=2+\left(D_{\mathrm{m}}-\mathrm{POM}-\mathrm{TEF}_{\mathrm{mmt}}\right) / \mathrm{TEF}
$$

Between-species comparisons required that $\delta^{13} \mathrm{C}$ values of marine mammal tissues be transformed to obtain $\delta^{13} \mathrm{C}$ of their average diet. This transformation was achieved by subtracting from $\delta^{13} \mathrm{C}$ value, the ${ }^{13} \mathrm{C}$ TEF of this particular tissue.
Table 2. Trophic enrichment factors (TEF) for ${ }^{13} \mathrm{C}$ and ${ }^{15} \mathrm{~N}$ in lipid-free tissues (blood red cells and serum, muscle and hair) of seals held in captivity on a constant diet of herring

\begin{tabular}{|lrrrl|}
\hline Tissue & $\mathrm{n}$ & $\begin{array}{c}\mathrm{TEF}{ }^{13} \mathrm{C} \\
(\%)\end{array}$ & $\begin{array}{c}\mathrm{TEF}^{15} \mathrm{~N} \\
(\%)\end{array}$ & Source \\
\hline Cells & 17 & +1.8 & +1.8 & Lesage (1999) \\
Serum & 17 & +1.5 & +3.0 & Lesage (1999) \\
Muscle & 2 & +1.3 & +2.4 & Hobson et al. (1996) \\
Hair & 2 & +2.3 & +2.3 & Lesage (1999) \\
\hline
\end{tabular}

The importance of different prey in marine mammals diet was examined using a 2-source mixing model:

$$
\%_{\mathrm{x}}=\left(\delta^{15} \mathrm{~N}_{\mathrm{c}}-\delta^{15} \mathrm{~N}_{\mathrm{y}}\right) /\left(\delta^{15} \mathrm{~N}_{\mathrm{x}}-\delta^{15} \mathrm{~N}_{\mathrm{y}}\right)
$$

where $\%_{\mathrm{x}}=$ proportion of the diet derived from prey ' $\mathrm{x}^{\prime}, \delta^{15} \mathrm{~N}_{\mathrm{c}}=$ nitrogen isotopic signature of the consumer's tissue, and $\delta^{15} \mathrm{~N}_{\mathrm{x}}$ and $\delta^{15} \mathrm{~N}_{\mathrm{y}}=$ nitrogen isotopic signatures of the consumer's tissue corresponding to exclusive diets of Types ' $x$ ' and ' $y$ ', respectively (Gearing 1991). In these models, the reliability of conclusions on prey contributions to the diet decreases with the increasing similarity of stable isotope values of prey.

Statistical analyses. All comparisons among and within groups were made using red blood cells or muscle tissue. For each marine mammal species-locationtissue combination, the effects of year, age, and sex on $\delta^{13} \mathrm{C}$ or $\delta^{15} \mathrm{~N}$ values (or $\delta^{13} \mathrm{C}$ of diet or TP), or any interaction between these effects were tested simultaneously using a multivariate analysis of variance (MANOVA), Type III sum of squares and the Hotelling's $T^{2}$ (2 groups) or Wilks' lambda ( $\geq 3$ groups) statistics (Hair et al. 1995). When no significant interaction between effects was indicated (i.e. p > 0.05), results from the n-way ANOVAs were examined for each isotope and effect. Based on these results, sex/ age/year groups of a given species/location identified as significantly different in their $\delta^{13} \mathrm{C}$ or $\delta^{15} \mathrm{~N}$ values were used for the between-species comparisons of isotopic signatures. For the latter analyses, the 2 isotopes were examined separately (i.e. two 1-way ANOVAs), since groups having significantly different $\delta^{13} \mathrm{C}$ values were not necessarily those that had significantly different $\delta^{15} \mathrm{~N}$ values. Post hoc Tukey's tests (1-way MANOVA or ANOVA) or Student's t-tests using leastsquares means (2- or 3-way MANOVA or ANOVA) were used to identify significant differences between pairs of values. Significance levels in each post hoc comparison were adjusted to account for the number of possible comparisons. A natural-log or square-root transformation was used when non-normality or variance-covariance matrix heterogeneity was indicated. When all of the standard parametric assumptions were still not met or when residuals were not normally dis- 
tributed, results from the previous analysis were validated by repeating the MANOVA or ANOVA using ranks for $\delta^{13} \mathrm{C}$ and $\delta^{15} \mathrm{~N}$ values (Conover 1980). Although a lack of significant differences may have resulted in some cases from small sample sizes, animals from a given species/group showing no significant differences between years in either isotope were grouped for subsequent analyses.

Differences among tissues in the estimated $\delta^{13} \mathrm{C}$ of average diet or TP were examined using paired $t$-tests. Harbour seals were segregated into 2 groups based on the date of sampling, i.e. 'early summer' (before July; $\mathrm{n}=30$ ) or 'late summer' (in or after July; $\mathrm{n}=$ 45) to identify potential movements outside the Lower Estuary during winter and spring from the comparison of blood cells and serum isotopic signatures. No such segregation was possible for Estuary grey seals due to the small number of captures for each sex and age class in the first period (all $\mathrm{n} \leq 3$ ). Diet composition of harbour and grey seals were assumed to be consistent between years, and thus years were grouped for each species, age class and sex to increase sample sizes. Year grouping increased the power of the test by increasing sample sizes, but at the same time it reduced the chances of detecting movements outside the Lower Estuary that might have happened in only 1 of 2 yr. Significance level for comparisons was fixed at 0.05 unless otherwise stated.

\section{RESULTS}

\section{Patterns of $\delta^{13} \mathrm{C}$ and $\delta^{15} \mathrm{~N}$ signatures}

The geographical location of collections significantly affected isotopic signatures of community components other than marine mammals (1-way MANOVA on ranks, $F_{2,509}=24.91 ; \mathrm{p}<$ 0.0001). Specifically, the location of collections had significant effects on $\delta^{13} \mathrm{C}$ values (ANOVA, $F_{1,510}=47.61$; $\mathrm{p}<0.0001$ ), but not on $\delta^{15} \mathrm{~N}$ values (ANOVA, $F_{1,510}=0.12 ; \mathrm{p}=0.72$ ). Com-
Table 3. Stable carbon and nitrogen isotope ratios and trophic position of components of St Lawrence Estuary community. Length classes (mm) are presented in parentheses after species names

\begin{tabular}{|c|c|c|c|c|}
\hline Species & $\mathrm{n}$ & $\begin{array}{c}\text { Mean } \delta^{13} \mathrm{C} \\
(\% o)\end{array}$ & $\begin{array}{c}\text { Mean } \delta^{15} \mathrm{~N} \\
(\% \text { o })\end{array}$ & $\begin{array}{l}\text { Trophic } \\
\text { position }\end{array}$ \\
\hline Particulate organic matter & 3 & $-22.9 \pm 1.7$ & $4.5 \pm 0.9$ & 1.0 \\
\hline $\begin{array}{l}\text { Benthic macroalgae } \\
\text { Ascophyllum nodosum } \\
\text { Laminaria longicruris }\end{array}$ & $\begin{array}{l}1 \\
1\end{array}$ & $\begin{array}{l}-17.6 \\
-13.8\end{array}$ & $\begin{array}{l}7.6 \\
7.9\end{array}$ & \\
\hline $\begin{array}{l}\text { Arthropoda - Crustacea } \\
\text { Copepoda } \\
\text { Calanus hyperboreus } \\
\text { Euchaeta norvegica } \\
\text { Microcalanus pusillus } \\
\text { Calanus sp. } \\
\text { Metridia sp. }\end{array}$ & $\begin{array}{l}1 \\
2 \\
1 \\
1 \\
2\end{array}$ & $\begin{array}{c}-21.2 \\
-19.8 \pm 0.2 \\
-18.9 \\
-19.6 \\
-20.4 \pm 0.2\end{array}$ & $\begin{array}{c}9.8 \\
10.5 \pm 0.4 \\
10.5 \\
10.9 \\
12.3 \pm 1.1\end{array}$ & $\begin{array}{l}2.5 \\
2.8 \\
2.8 \\
2.9 \\
3.3\end{array}$ \\
\hline $\begin{array}{l}\text { Malacostraca } \\
\text { Mysidacea } \\
\text { Mysis sp. }\end{array}$ & 1 & -20.4 & 10.9 & 2.9 \\
\hline $\begin{array}{l}\text { Amphipoda } \\
\text { Caprella sp. } \\
\text { Gammarus sp. }\end{array}$ & $\begin{array}{l}1 \\
1\end{array}$ & $\begin{array}{l}-17.9 \\
-17.8\end{array}$ & $\begin{array}{l}6.0 \\
9.1\end{array}$ & $\begin{array}{l}1.4 \\
2.4\end{array}$ \\
\hline $\begin{array}{l}\text { Euphausiacea } \\
\text { Thysanoessa inermis and T. raschii } \\
\text { Meganyctiphanes norvegica }\end{array}$ & $\begin{array}{l}4 \\
1\end{array}$ & $\begin{array}{c}-19.6 \pm 1.1 \\
-19.3\end{array}$ & $\begin{array}{c}10.1 \pm 1.5 \\
11.5\end{array}$ & $\begin{array}{l}2.6 \\
3.1\end{array}$ \\
\hline $\begin{array}{l}\text { Decapoda }^{\text {a }} \\
\text { Rock crab Cancer irroratus (176-256) } \\
\text { Pandalus borealis } \\
\text { Snow crab Chionoecetes opilio (60) } \\
\text { Pandalus montagui } \\
\text { Snow crab Chionoecetes opilio (910) } \\
\text { Sclerocrangon boreas }\end{array}$ & $\begin{array}{l}2 \\
1 \\
1 \\
2 \\
3 \\
2\end{array}$ & $\begin{array}{l}-16.5 \pm 0.4 \\
-17.8 \\
-17.8 \\
-17.5 \pm 0.4 \\
-16.7 \pm 0.6 \\
-17.8 \pm 0.6\end{array}$ & $\begin{array}{c}12.7 \pm 1.0 \\
13.2 \\
13.2 \\
13.8 \pm 0.0 \\
14.0 \pm 0.7 \\
14.2 \pm 0.2\end{array}$ & $\begin{array}{l}3.4 \\
3.5 \\
3.5 \\
3.7 \\
3.8 \\
3.9\end{array}$ \\
\hline $\begin{array}{l}\text { Mollusca } \\
\text { Bivalvia } \\
\text { Blue mussel Mytilus edulis } \\
\text { Chlamys islandica }\end{array}$ & $\begin{array}{l}1 \\
1\end{array}$ & $\begin{array}{l}-19.2 \\
-16.7\end{array}$ & $\begin{array}{c}7.4 \\
11.8\end{array}$ & $\begin{array}{l}1.8 \\
3.1\end{array}$ \\
\hline $\begin{array}{l}\text { Annelida - Polychaeta } \\
\quad \text { Nereis sp. }\end{array}$ & 1 & -16.2 & 10.6 & 2.8 \\
\hline $\begin{array}{l}\text { Echinodermata } \\
\text { Echinoidea } \\
\text { Green urchin } \\
\text { Strongylocentrotus droebachiensis }\end{array}$ & 3 & $-14.6 \pm 0.2$ & $7.7 \pm 0.7$ & 2.0 \\
\hline $\begin{array}{l}\text { Pisces }^{\mathrm{b}} \\
\text { American sand lance } \\
\text { Ammodites americanus (96-102) }\end{array}$ & 2 & $-19.9 \pm 0.4$ & $11.5 \pm 0.3$ & 3.0 \\
\hline $\begin{array}{l}\text { American sand lance } \\
\text { Ammodites americanus (115-205) }\end{array}$ & 9 & $-18.5 \pm 0.4$ & $11.4 \pm 0.3$ & 3.0 \\
\hline $\begin{array}{l}\text { Atlantic herring Clupea } \\
\text { harengus harengus (103-165) }\end{array}$ & 10 & $-18.8 \pm 1.1$ & $13.1 \pm 0.6$ & 3.5 \\
\hline $\begin{array}{l}\text { Greenland halibut Reinhardtius } \\
\text { hippoglossoides (135-165) }\end{array}$ & $\begin{array}{r}5 \\
13\end{array}$ & $-19.7 \pm 0.4$ & $13.1 \pm 0.7$ & 3.5 \\
\hline $\begin{array}{l}\text { American plaice Hippoglossoides } \\
\text { platessoides (184-206) }\end{array}$ & 3 & $-18.0 \pm 0.1$ & $13.7 \pm 0.2$ & 3.7 \\
\hline Pollock Pollachius virens (327) & 1 & -19.7 & 13.8 & 3.7 \\
\hline Redfish Sebastes sp. (79-126) & 6 & $-19.1 \pm 0.7$ & $13.9 \pm 0.5$ & 3.8 \\
\hline Redfish Sebastes sp. (185-305) & 6 & $-18.9 \pm 0.3$ & $14.0 \pm 0.3$ & 3.8 \\
\hline $\begin{array}{l}\text { Atlantic herring Clupea } \\
\text { harengus harengus (215-315) }\end{array}$ & 14 & $-18.9 \pm 0.7$ & $13.9 \pm 0.4$ & 3.8 \\
\hline Atlantic cod Gadus morhua (230) & 1 & -18.4 & 14.0 & 3.8 \\
\hline Arctic cod Boreogadus saida (98) & 1 & -18.2 & 14.2 & 3.9 \\
\hline $\begin{array}{l}\text { Greenland halibut Reinhardtius } \\
\text { hippoglossoides (219-275) }\end{array}$ & 8 & $-19.2 \pm 0.3$ & $14.3 \pm 0.2$ & 3.9 \\
\hline
\end{tabular}


Table 3 (continued)

\begin{tabular}{|c|c|c|c|c|}
\hline Species & $\mathrm{n}$ & $\begin{array}{c}\text { Mean } \delta^{13} \mathrm{C} \\
(\%)\end{array}$ & $\begin{array}{c}\text { Mean } \delta^{15} \mathrm{~N} \\
(\%)\end{array}$ & $\begin{array}{l}\text { Trophic } \\
\text { position }\end{array}$ \\
\hline Cottidae (196-205) & 2 & $-16.7 \pm 0.9$ & $14.4 \pm 0.4$ & 3.9 \\
\hline $\begin{array}{l}\text { Lumpfish Cyclopterus lumpus } \\
(176-320)\end{array}$ & 6 & $-19.5 \pm 0.5$ & $14.6 \pm 0.3$ & 4.0 \\
\hline Skate Raja sp. (126-330) & 5 & $-18.0 \pm 0.5$ & $14.6 \pm 0.6$ & 4.0 \\
\hline $\begin{array}{l}\text { Fourline snakeblenny } \\
\text { Eumesogrammus praecisus (300) }\end{array}$ & 2 & $-18.2 \pm 0.0$ & $14.7 \pm 0.6$ & 4.0 \\
\hline $\begin{array}{l}\text { Witch flounder Glyptocephalus } \\
\text { cynoglossus }(190-460)\end{array}$ & 19 & $-17.6 \pm 0.5$ & $14.7 \pm 0.5$ & 4.0 \\
\hline $\begin{array}{l}\text { Rainbow smelt Osmerus mordax } \\
(78-99)\end{array}$ & 2 & $-20.0 \pm 0.2$ & $14.8 \pm 0.4$ & 4.0 \\
\hline $\begin{array}{l}\text { Arctic cod Boreogadus saida } \\
(140-146)\end{array}$ & 2 & $-18.0 \pm 0.2$ & $14.8 \pm 0.2$ & 4.0 \\
\hline $\begin{array}{l}\text { American plaice } \\
\text { Hippoglossoides platessoides } \\
(232-315)\end{array}$ & 6 & $-18.2 \pm 0.5$ & $14.9 \pm 0.4$ & 4.1 \\
\hline Snailfish Liparis sp. (184-235) & 4 & $-18.0 \pm 0.4$ & $14.9 \pm 0.4$ & 4.1 \\
\hline Lycodes sp. $(215-340)$ & 3 & $-18.1 \pm 1.0$ & $15.0 \pm 1.1$ & 4.1 \\
\hline $\begin{array}{l}\text { Atlantic tomcod } \\
\text { Microgadus tomcod (88-107) }\end{array}$ & 3 & $-16.2 \pm 1.6$ & $15.0 \pm 0.2$ & 4.1 \\
\hline Macrouridae $(230)$ & 1 & -18.7 & 15.0 & 4.1 \\
\hline $\begin{array}{l}\text { American eel } \\
\text { Anguilla rostrata (715-1040) }\end{array}$ & 18 & $-21.1 \pm 2.9$ & $15.1 \pm 1.4$ & 4.1 \\
\hline $\begin{array}{l}\text { Greenland halibut Reinhardtius } \\
\text { hippoglossoides }(344-420)\end{array}$ & 6 & $-18.6 \pm 0.3$ & $15.3 \pm 0.8$ & 4.2 \\
\hline $\begin{array}{l}\text { American plaice Hippoglossoides } \\
\text { platessoides }(335-410)\end{array}$ & 4 & $-18.1 \pm 0.1$ & $15.4 \pm 0.2$ & 4.2 \\
\hline $\begin{array}{l}\text { Smooth flounder } \\
\text { Liopsetta putnami (76-176) }\end{array}$ & 3 & $-14.3 \pm 0.8$ & $15.4 \pm 0.4$ & 4.2 \\
\hline $\begin{array}{l}\text { Atlantic cod Gadus morhua } \\
(330-545)\end{array}$ & 6 & $-18.3 \pm 0.5$ & $15.6 \pm 0.7$ & 4.3 \\
\hline $\begin{array}{l}\text { Fourbeard rockling } \\
\text { Enchelyopus cimbrius (235) }\end{array}$ & 1 & -18.9 & 15.6 & 4.3 \\
\hline $\begin{array}{l}\text { Atlantic tomcod } \\
\text { Microgadus tomcod (145-190) }\end{array}$ & 6 & $-18.0 \pm 1.6$ & $15.7 \pm 0.4$ & 4.3 \\
\hline $\begin{array}{l}\text { Black dogfish } \\
\text { Centroscyllium fabricii (405-455) }\end{array}$ & 3 & $-18.4 \pm 0.1$ & $15.7 \pm 0.5$ & 4.3 \\
\hline $\begin{array}{l}\text { Rainbow smelt } \\
\text { Osmerus mordax (152-216) }\end{array}$ & 7 & $-19.0 \pm 0.4$ & $15.7 \pm 0.4$ & 4.3 \\
\hline Cottidae $(280)$ & 1 & -17.4 & 15.8 & 4.3 \\
\hline $\begin{array}{l}\text { Atlantic tomcod } \\
\text { Microgadus tomcod (270-330) }\end{array}$ & 4 & $-18.1 \pm 0.2$ & $16.4 \pm 0.1$ & 4.5 \\
\hline $\begin{array}{l}\text { Atlantic poacher Agonus decagonus } \\
(205)\end{array}$ & 1 & -18.4 & 16.2 & 4.5 \\
\hline $\begin{array}{l}\text { Smooth flounder } \\
\text { Liopsetta putnami (316-334) }\end{array}$ & 3 & $-14.8 \pm 0.5$ & $16.5 \pm 0.4$ & 4.5 \\
\hline $\begin{array}{l}\text { White hake Urophycis tenuis } \\
(324-520)\end{array}$ & 3 & $-17.8 \pm 0.3$ & $16.7 \pm 0.5$ & 4.6 \\
\hline
\end{tabular}

erican plaice, Arctic cod, Atlantic cod, Atlantic herring, lumpfish, the Cottidae (sculpins) and Rajidae (skates) ${ }^{1}$. Consequently, regions were considered separately in subsequent analyses that involved either $\mathrm{C}$ or both isotopes.

Isotopic signatures varied with size in several fish species, and thus length classes were analysed separately (Tables $3 \& 4$ ). In all cases where size had a significant effect on $\delta^{13} \mathrm{C}$ (i.e. Gulf Atlantic herring, mackerel, witch flounder, pollock, redfish and Greenland halibut, and Estuary American sand lance, Greenland halibut and rainbow smelt ${ }^{1}$ ) or $\delta^{15} \mathrm{~N}$ (i.e. Atlantic cod, herring, tomcod and mackerel, American plaice, Arctic cod, Greenland halibut, the Cottidae, lumpfish, rainbow smelt, redfish, white hake, and smooth flounder ${ }^{1}$ ), fishes were enriched in ${ }^{13} \mathrm{C}$ or ${ }^{15} \mathrm{~N}$ as they increased in length.

Significant variations in isotopic signatures were observed between years in all marine mammal groups sampled during more than 1 yr except Estuary grey seals (3-way MANOVA, $F_{2,10}=$ 0.98 for effect of year, $p=0.41$ ). In general, a higher enrichment in either one or both isotopes was observed in 1995 compared to subsequent years. Stable $\mathrm{N}$ isotope ratios remained consistent between years in 2+ and yearlings of harbour seals and in beluga whales. However, $\delta^{13} \mathrm{C}$ was higher in yearling and 2+ harbour seals from 1995 than in those from 1996 or 1997 (post hoc $t$-tests: $\mathrm{p}<0.005$ for each comparison) and in beluga whales from 1997 compared to 1988, 1989 or 1990 (post hoc $t$-tests, each $\mathrm{p} \leq 0.001)$. Female hooded seals had similar ${ }^{15} \mathrm{~N}$ enrichment in 1995 and 1996 (post hoc $t$-test, p = 0.565), but this was not the case for male hooded seals, whose ${ }^{15} \mathrm{~N}$-enrichment was greater in 1995 than in 1996 (post hoc $t$-test, $\mathrm{p}<0.001$ ). Both male and female hooded seals were more ${ }^{13} \mathrm{C}$-enriched in 1995 than in 1996 (2-way MANOVA, ANOVA for effect of year on $\delta^{13} C_{1} F_{1,40}=4.52 ; p=0.040$ ). Male and female Gulf harp seals captured in 1995 were more

\footnotetext{
${ }^{1}$ Corresponding scientific names are presented in Tables $3 \& 4$

munity components collected in the Gulf were depleted in ${ }^{13} \mathrm{C}(\bar{x} \pm \mathrm{SE}=-19.2 \% \pm 0.1)$ relative to those from the Estuary $(\bar{x} \pm \mathrm{SE}=-18.6 \% \pm 0.1)$, but $\delta^{15} \mathrm{~N}$ values were similar in the 2 regions $(\bar{X} \pm \mathrm{SE}=13.8 \% \pm 0.1$ and $14.1 \%$ \pm 0.1 for the Gulf and Estuary, respectively). This pattern was also observed when examining fish species individually, particularly the American sand lance, Am- 
enriched in ${ }^{15} \mathrm{~N}$ than those captured in 1996 (2-way MANOVA, ANOVA for effect of year on $\delta^{13} \mathrm{~N}, F_{1,35}=5.96 ; \mathrm{p}=$ 0.020 ), but their $\delta^{13} \mathrm{C}$ signatures were similar during the 2 years $\left(F_{1,35}=1.59\right.$; $\mathrm{p}=0.215)$.

Tissue $\delta^{13} \mathrm{C}$ and $\delta^{15} \mathrm{~N}$ varied with age in marine mammals. This was most evident in the 1995 harbour seal samples, which included nursing and weaned pups, yearlings and 2+ individuals, and in 4 of 5 individually marked harbour seals recaptured 2 to 3 times over periods ranging from 1 to 12 mo. During nursing, harbour seal pup red blood cells had similar $\delta^{13} \mathrm{C}$ values to other age classes, but were more enriched in ${ }^{15} \mathrm{~N}$ than older individuals (post hoc $t$-tests significant, i.e. $\mathrm{p}<0.008$, for nursing pups vs each of $2+$ individuals, yearlings and weaned pups) (Fig. 2a). $\delta^{15} \mathrm{~N}$ values decreased in pups during their first year of life, reaching a minimum at about Age 1. In the 1995 harbour seals, yearlings and $2+$ individuals had similar $\delta^{13} \mathrm{C}$ and $\delta^{15} \mathrm{~N}$ values. However, in 1996 when only yearlings and 2+ individuals were sampled, yearlings were significantly depleted in both ${ }^{13} \mathrm{C}$ and ${ }^{15} \mathrm{~N}$ relative to older seals (post hoc $t$-tests, both $\mathrm{p} \leq 0.001$ ). Similarly, muscle tissues of harp seal yearlings captured in the Estuary were significantly depleted in ${ }^{13} \mathrm{C}$ relative to those of $2+$ individuals (2-way MANOVA, ANOVA for effect of age on $\delta^{13} \mathrm{C}, F_{1,14}=25,17 ; \mathrm{p}=0.0002$ ), but their mean $\delta^{15} \mathrm{~N}$ were not significantly different from those of $2+$ individuals $\left(F_{1,14}=0.36 ; \mathrm{p}=0.56\right)$ (Fig. $\left.2 \mathrm{~b}\right)$. This general pattern also prevailed in both blood red cells and serum in 4 of 5 harbour seals recaptured several times, although these changes were usually 2 to 3 times larger in serum than in red cells (Fig. 3). Isotopic signatures in red blood cells of 2+ grey seals from the Estuary varied differently between years relative to yearlings, resulting in a significant interaction between year and age class. In 1995, mean $\delta^{13} \mathrm{C}$ and $\delta^{15} \mathrm{~N}$ values were slightly lower in yearlings $(n=4)$ compared to $2+$ animals $(\mathrm{n}=3)$, but these differences were not statistically significant (post
Table 4. Stable carbon and nitrogen isotope ratios and trophic position of components of Gulf of St Lawrence community. Length classes (mm) are presented in parentheses after species names

\begin{tabular}{|c|c|c|c|c|}
\hline Species & $\mathrm{n}$ & $\underset{(\% \circ)}{\operatorname{Mean} \delta^{13} \mathrm{C}}$ & $\underset{(\%)}{\operatorname{Mean} \delta^{15} \mathrm{~N}}$ & $\begin{array}{l}\text { Trophic } \\
\text { position }\end{array}$ \\
\hline \multicolumn{5}{|l|}{ Arthropoda-Crustacea } \\
\hline \multicolumn{5}{|l|}{ Decapoda } \\
\hline $\begin{array}{l}\text { Lobster Homarus americanus } \\
(125-200)^{\mathrm{a}}\end{array}$ & 6 & $-17.5 \pm 0.4$ & $11.4 \pm 0.4$ & 3.0 \\
\hline Snow crab Chionoecetes opilio $(107)^{\mathrm{b}}$ & 1 & -17.6 & 12.1 & 3.2 \\
\hline Eualus sp. -3 individuals & 1 & -18.0 & 12.6 & 3.4 \\
\hline Pasiphaea tarda & 1 & -19.3 & 13.0 & 3.5 \\
\hline $\begin{array}{l}\text { Snow crab Chionoecetes opilio } \\
(55-57)^{\mathrm{b}}\end{array}$ & 2 & $-18.7 \pm 0.1$ & $13.3 \pm 0.1$ & 3.6 \\
\hline Sclerocrangon boreas - 2 individuals & 1 & -18.5 & 13.3 & 3.6 \\
\hline Pasiphaea multidentata - 3 individuals & 2 & $-19.6 \pm 0.9$ & $13.5 \pm 0.1$ & 3.6 \\
\hline Pandalus montagui & 5 & $-18.0 \pm 1.1$ & $13.5 \pm 0.4$ & 3.6 \\
\hline Pandalus sp. & 4 & $-18.0 \pm 0.4$ & $13.9 \pm 0.5$ & 3.8 \\
\hline Lebbeus groenlandicus & 1 & -17.5 & 14.0 & 3.8 \\
\hline Sclerocrangon boreas & 1 & -16.4 & 14.8 & 4.0 \\
\hline \multicolumn{5}{|l|}{ Mollusca } \\
\hline \multicolumn{5}{|l|}{ Gasteropoda } \\
\hline Littorina sp. & 2 & $-14.8 \pm 0.1$ & $8.6 \pm 0.0$ & 2.2 \\
\hline Whelk Buccinum undatum (48) ${ }^{\mathrm{c}}$ & 3 & $-17.6 \pm 0.5$ & $11.1 \pm 0.3$ & 3.0 \\
\hline \multicolumn{5}{|l|}{ Bivalvia } \\
\hline Blue mussel Mytilus edulis & 1 & -19.6 & 7.3 & 1.8 \\
\hline & $13.0 \pm 0.4$ & 3.5 \\
\hline \multicolumn{5}{|l|}{ Pisces $^{\mathrm{e}}$} \\
\hline $\begin{array}{l}\text { Atlantic mackerel Scomber } \\
\text { scombrus (74-83) }\end{array}$ & 3 & $-21.0 \pm 0.3$ & $11.2 \pm 0.3$ & 3.0 \\
\hline $\begin{array}{l}\text { American sand lance Ammodites } \\
\text { americanus (147-215) }\end{array}$ & 8 & $-19.6 \pm 0.3$ & $11.9 \pm 0.5$ & 3.2 \\
\hline $\begin{array}{l}\text { Lumpfish Cyclopterus lumpus } \\
(125-136)\end{array}$ & 4 & $-20.9 \pm 0.8$ & $12.7 \pm 0.2$ & 3.4 \\
\hline $\begin{array}{l}\text { Greenland halibut Reinhardtius } \\
\text { hippoglossoides (165-185) }\end{array}$ & 2 & $-18.9 \pm 0.1$ & $12.8 \pm 0.1$ & 3.4 \\
\hline $\begin{array}{l}\text { Atlantic mackerel Scomber } \\
\text { scombrus }(220-403)\end{array}$ & 13 & $-20.2 \pm 0.5$ & $12.9 \pm 0.6$ & 3.5 \\
\hline $\begin{array}{l}\text { Winter flounder Pseudopleuronectes } \\
\text { americanus (205-345) }\end{array}$ & 6 & $-18.9 \pm 0.5$ & $13.0 \pm 0.4$ & 3.5 \\
\hline $\begin{array}{l}\text { White baraccudina Notolepis rissoi } \\
(220-245)\end{array}$ & 5 & $-19.8 \pm 0.3$ & $13.1 \pm 0.6$ & 3.5 \\
\hline $\begin{array}{l}\text { Atlantic herring Clupea harengus } \\
\text { harengus (144-180) }\end{array}$ & 6 & $-20.7 \pm 0.7$ & $13.3 \pm 0.4$ & 3.6 \\
\hline $\begin{array}{l}\text { Atlantic herring Clupea harengus } \\
\text { harengus (185-325) }\end{array}$ & 30 & $-19.9 \pm 0.3$ & $13.4 \pm 0.4$ & 3.6 \\
\hline Pollock Pollachius virens (230-290) & 4 & $-20.4 \pm 0.1$ & $13.6 \pm 0.9$ & 3.7 \\
\hline Capelin Mallotus villosus (105-155) & 13 & $-20.0 \pm 0.8$ & $13.7 \pm 0.7$ & 3.7 \\
\hline $\begin{array}{l}\text { Yellowtail flounder Limanda } \\
\text { ferruginea }(235-305)\end{array}$ & 4 & $-19.1 \pm 0.8$ & $13.7 \pm 0.4$ & 3.7 \\
\hline $\begin{array}{l}\text { Lumpfish Cyclopterus lumpus } \\
(260-265)\end{array}$ & 2 & $-20.5 \pm 0.4$ & $13.7 \pm 0.3$ & 3.7 \\
\hline $\begin{array}{l}\text { Longfin hake Urophycis chesteri } \\
(160-320)\end{array}$ & 5 & $-19.8 \pm 0.4$ & $14.0 \pm 0.5$ & 3.8 \\
\hline Arctic cod Boreogadus saida (121-135) & 6 & $-19.9 \pm 0.6$ & $14.0 \pm 0.2$ & 3.8 \\
\hline Greenland cod Gadus ogac (160-235) & 7 & $-19.3 \pm 0.8$ & $14.3 \pm 0.4$ & 3.9 \\
\hline $\begin{array}{l}\text { Greenland halibut Reinhardtius } \\
\text { hippoglossoides }(230-270)\end{array}$ & 4 & $-19.8 \pm 0.4$ & $14.3 \pm 0.3$ & 3.9 \\
\hline $\begin{array}{l}\text { American plaice Hippoglossoides } \\
\text { platessoides }(134-195)\end{array}$ & 4 & $-19.6 \pm 0.4$ & $14.3 \pm 0.4$ & 3.9 \\
\hline Pollock Pollachius virens (300-310) & 2 & $-19.6 \pm 0.4$ & $14.4 \pm 0.1$ & 3.9 \\
\hline
\end{tabular}


Table 4 (continued)

\begin{tabular}{|c|c|c|c|c|}
\hline Species & $\mathrm{n}$ & $\underset{(\% o)}{\operatorname{Mean} \delta^{13} \mathrm{C}}$ & $\underset{(\% o)}{\operatorname{Mean} \delta^{15} \mathrm{~N}}$ & $\begin{array}{l}\text { Trophic } \\
\text { position }\end{array}$ \\
\hline $\begin{array}{l}\text { American plaice Hippoglossoides } \\
\text { platessoides }(220-320)\end{array}$ & 8 & $-19.4 \pm 0.5$ & $14.5 \pm 0.4$ & 3.9 \\
\hline Lycodes sp. (185-365) & 6 & $-19.2 \pm 0.8$ & $14.5 \pm 0.4$ & 3.9 \\
\hline Atlantic cod Gadus morhua (145-320) 1 & 11 & $-19.5 \pm 0.5$ & $14.6 \pm 0.8$ & 4.0 \\
\hline Redfish Sebastes sp. (120-145) & 5 & $-20.1 \pm 0.1$ & $14.6 \pm 0.4$ & 4.0 \\
\hline Redfish Sebastes sp. (152-335) & 11 & $-19.2 \pm 0.5$ & $14.7 \pm 0.7$ & 4.0 \\
\hline $\begin{array}{l}\text { Witch flounder Glyptocephalus } \\
\text { cynoglossus (165-190) }\end{array}$ & 3 & $-19.4 \pm 0.2$ & $14.6 \pm 0.3$ & 4.0 \\
\hline $\begin{array}{l}\text { Haddock Melanogrammus aeglefinus } \\
\text { (185) }\end{array}$ & 1 & -19.6 & 14.7 & 4.0 \\
\hline Skate Raja sp. (240-405) & 4 & $-18.7 \pm 0.2$ & $14.8 \pm 0.3$ & 4.0 \\
\hline Silver hake Merluccius bilinearis (365) & 1 & -19.6 & 14.8 & 4.0 \\
\hline $\begin{array}{l}\text { Moustache sculpin Triglops murrayi } \\
(145-175)\end{array}$ & 3 & $-19.6 \pm 0.1$ & $15.0 \pm 0.3$ & 4.1 \\
\hline Macrouridae (168-300) & 4 & $-19.1 \pm 0.9$ & $15.1 \pm 0.6$ & 4.1 \\
\hline Spatulate sculpin Icelus spatula (194) & 1 & -18.6 & 15.2 & 4.1 \\
\hline Cottidae $(210-265)$ & 3 & $-18.8 \pm 0.5$ & $15.2 \pm 0.1$ & 4.1 \\
\hline Arctic cod Boreogadus saida (150-220) & 6 & $-19.4 \pm 0.4$ & $15.2 \pm 0.6$ & 4.1 \\
\hline $\begin{array}{l}\text { White hake Urophycis tenuis } \\
(215-245)\end{array}$ & 3 & $-17.5 \pm 0.3$ & $15.2 \pm 0.3$ & 4.1 \\
\hline $\begin{array}{l}\text { Witch flounder Glyptocephalus } \\
\text { cynoglossus (285-370) }\end{array}$ & 5 & $-17.9 \pm 0.5$ & $15.2 \pm 0.6$ & 4.1 \\
\hline $\begin{array}{l}\text { Greenland halibut Reinhardtius } \\
\text { hippoglossoides (305-430) }\end{array}$ & 6 & $-18.7 \pm 0.8$ & $15.3 \pm 0.6$ & 4.2 \\
\hline $\begin{array}{l}\text { White hake Urophycis tenuis } \\
(315-525)\end{array}$ & 4 & $-18.4 \pm 0.5$ & $15.7 \pm 0.8$ & 4.3 \\
\hline $\begin{array}{l}\text { Black dogfish Centroscyllium fabricii } \\
(300-395)\end{array}$ & 3 & $-18.6 \pm 0.2$ & $15.7 \pm 0.2$ & 4.3 \\
\hline Arctic cod Boreogadus saida (240-280) & 2 & $-19.5 \pm 0.1$ & $15.8 \pm 0.3$ & 4.3 \\
\hline $\begin{array}{l}\text { American plaice Hippoglossoides } \\
\text { platessoides }(360-385)\end{array}$ & 3 & $-18.8 \pm 0.0$ & $15.8 \pm 0.7$ & 4.3 \\
\hline Atlantic cod Gadus morhua (330-466) & 9 & $-19.2 \pm 0.4$ & $15.8 \pm 0.7$ & 4.3 \\
\hline $\begin{array}{l}\text { Atlantic wolffish Anarhichas lupus } \\
(230-355)\end{array}$ & 4 & $-18.2 \pm 0.6$ & $15.9 \pm 0.5$ & 4.4 \\
\hline $\begin{array}{l}\text { Fourbeard rockling Enchelyopus } \\
\text { cimbrius (195-220) }\end{array}$ & 3 & $-19.3 \pm 0.2$ & $16.0 \pm 0.3$ & 4.4 \\
\hline $\begin{array}{l}\text { Cottidae }(280-301) \\
\text { Sea raven Hemitripterus }\end{array}$ & 4 & $-18.2 \pm 0.9$ & $16.1 \pm 0.2$ & 4.4 \\
\hline americanus (200-385) & 3 & $-18.3 \pm 0.1$ & $16.3 \pm 0.8$ & 4.5 \\
\hline Redfish Sebastes sp. (370-415) & 3 & $-18.5 \pm 0.2$ & $16.6 \pm 0.7$ & 4.5 \\
\hline $\begin{array}{l}\text { Fourline snakeblenny Eumeso- } \\
\text { grammus praecisus (145-165) }\end{array}$ & 3 & $-18.3 \pm 0.3$ & $16.8 \pm 0.1$ & 4.6 \\
\hline Cottidae $(320-327)$ & 2 & $-18.5 \pm 0.3$ & $17.0 \pm 0.0$ & 4.7 \\
\hline $\begin{array}{l}{ }^{\mathrm{a}} \text { Length of cephalothorax }(\mathrm{mm}) \\
\mathrm{b}^{\mathrm{b}} \text { Width of carapace }(\mathrm{mm}) \\
{ }^{\mathrm{c}} \text { Length of shell }(\mathrm{mm}) \\
{ }^{\mathrm{d}} \text { Length of mantle }(\mathrm{mm}) \\
{ }^{\mathrm{e}} \text { Fork lengths }(\mathrm{mm})\end{array}$ & & & & \\
\hline
\end{tabular}

Sex influenced the $\delta^{15} \mathrm{~N}$ signature of $2+$ animals, but not that of yearlings. Males of Age 2+ were generally ${ }^{15} \mathrm{~N}$ enriched relative to $2+$ females (Table 5). This effect was significant in beluga whales (post hoc $t$-tests, $\mathrm{p} \leq$ 0.003 in each year) and in hooded and harp seals captured in the Gulf in 1995 (post hoc $t$-tests, $\mathrm{p}=0.004$ and 0.01 , respectively), and marginally insignificant in harp (post hoc $t$-test: $\mathrm{p}=0.085$ ) and grey seals $(p=0.072)$ captured in the Gulf in 1996. Nitrogen isotope ratios were similar in both sexes of harbour seals, Estuary grey or harp seals, and in hooded seals captured in 1996. In the latter 2 groups, $\delta^{15} \mathrm{~N}$ values were 0.2 to $0.3 \%$ lower in males than in females.

Stable carbon isotope ratios differed between sexes only in $2+$ animals, and only in 2 species (Table 5 ). In beluga whales, females from each of the $4 \mathrm{yr}$ of sampling were consistently depleted in ${ }^{13} \mathrm{C}$ relative to males (2-way MANOVA, ANOVA for effect of sex on $\delta^{13} C_{1} F_{1,29}=62.06, p=0.0001$ ). Contradictory results were obtained from harbour seals between years, since females were significantly ${ }^{13} \mathrm{C}$-enriched relative to males in 1995 (post hoc $t$-test: $\mathrm{p}=0.0005$ ), whereas the 2 sexes had similar enrichments in 1996 and 1997 (post hoc $t$-test: $\mathrm{p}=0.717$ and 0.888 , respectively).

\section{Trophic structure of St Lawrence community}

Carbon isotope ratios varied by up to $9 \%$ among organisms from a given region (Fig. 4, Tables 3 \& 4). Primary producers were found at both extremes, with macroalgae being the most ${ }^{13} \mathrm{C}$-enriched source, and POM the least ${ }^{13} \mathrm{C}$-enriched source. Given the wide variation in $\delta^{13} \mathrm{C}$ values

hoc $t$-tests: $\mathrm{p}=0.060$ and 0.048 , respectively). In 1996 , $\delta^{13} \mathrm{C}$ and $\delta^{15} \mathrm{~N}$ signatures of $2+$ individuals $(\mathrm{n}=6)$ were lower than in 1995, and were similar to those of yearlings from $1996(\mathrm{n}=6)$ (post hoc $t$-tests: $\mathrm{p}=0.916$ and 0.251 for $\delta^{13} \mathrm{C}$ and $\delta^{15} \mathrm{~N}$, respectively). Small sample sizes may have been responsible for the shifting patterns. among organisms from a given region and TP, 2 main C pathways appear to be important in the St Lawrence food webs (Fig. 4). Each pathway incorporates a progressive enrichment of $\sim 1.5 \%$ in $\delta^{13} \mathrm{C}$ with trophic level. Assuming that the 2 to $3 \%$ depletion of Gulf POM relative to Estuary POM would translate into a similar depletion in Gulf zooplankton, ${ }^{13} \mathrm{C}$ contents 

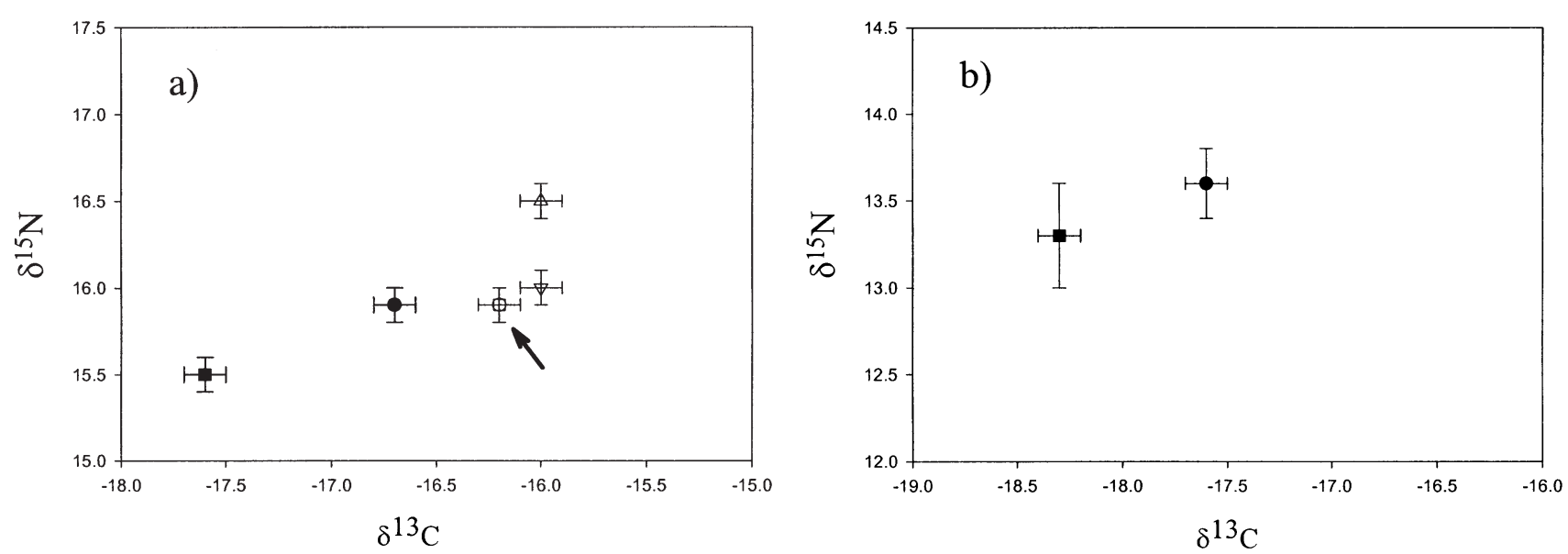

Fig. 2. Isotopic signature (\%o $\pm \mathrm{SE})$ of $2+$ individuals $(\bullet, 0)$, yearlings $(\boldsymbol{\square}, \square)$, weaned pups $(\boldsymbol{\nabla}, \nabla)$, and nursing pups $(\boldsymbol{\Lambda}, \Delta)$ of $(\mathrm{a})$ red blood cells of harbour seals Phoca vitulina and (b) muscle tissues of Estuary harp seals Phoca groenlandica from 1995 (open symbols) and 1996 (filled symbols). Arrow indicates superimposition of 2 symbols
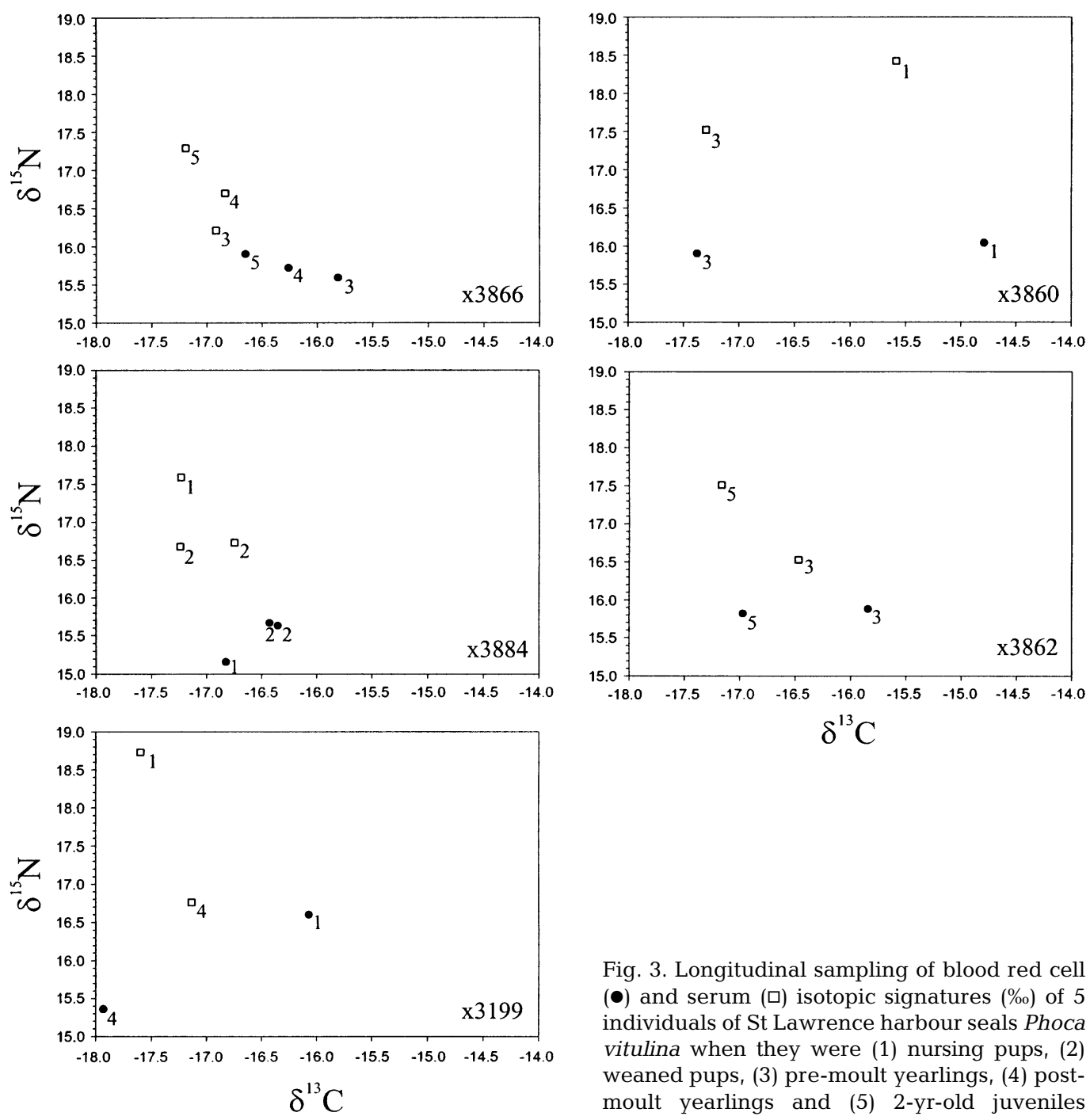

Fig. 3. Longitudinal sampling of blood red cell $(\bullet)$ and serum () isotopic signatures (\%) of 5 individuals of St Lawrence harbour seals Phoca vitulina when they were (1) nursing pups, (2) weaned pups, (3) pre-moult yearlings, (4) postmoult yearlings and (5) 2-yr-old juveniles 
Table 5. Mean trophic position (TP $\pm \mathrm{SD})$, and carbon $\left(\delta^{13} \mathrm{C}\right)$ and nitrogen $\left(\delta^{15} \mathrm{~N}\right)$ isotope ratios for male and female yearling and $2+$ marine mammals from Estuary and Gulf of St Lawrence

\begin{tabular}{|c|c|c|c|c|c|c|c|c|c|c|}
\hline $\begin{array}{l}\text { Species } \\
\text { Region }\end{array}$ & Year & $\begin{array}{l}\text { Age } \\
\text { class }\end{array}$ & $\mathrm{n}$ & $\delta^{13} \mathrm{C}$ & $\begin{array}{l}\text { Iale } \\
\qquad \delta^{15} \mathrm{~N}\end{array}$ & $\mathrm{TP}$ & $\mathrm{n}$ & $\delta^{13} \mathrm{C}$ & $\begin{array}{l}\text { nale } \\
\qquad \delta^{15} \mathrm{~N}\end{array}$ & $\mathrm{TP}$ \\
\hline \multicolumn{11}{|l|}{ Harbour seal } \\
\hline \multirow[t]{4}{*}{ Estuary } & 1995 & Yearling & 4 & $-16.3 \pm 0.4$ & $16.0 \pm 0.2$ & $4.9 \pm 0.1$ & 6 & $-16.2 \pm 0.4$ & $15.8 \pm 0.2$ & $4.8 \pm 0.1$ \\
\hline & & $2+$ individuals & 12 & $-16.4 \pm 0.5$ & $15.9 \pm 0.4$ & $4.8 \pm 0.1$ & 6 & $-15.7 \pm 0.5$ & $15.9 \pm 0.2$ & $4.8 \pm 0.1$ \\
\hline & $1996-97$ & Yearling & 13 & $-17.6 \pm 0.5$ & $15.6 \pm 0.5$ & $4.8 \pm 0.2$ & 10 & $-17.5 \pm 0.3$ & $15.3 \pm 0.7$ & $4.7 \pm 0.2$ \\
\hline & & $2+$ individuals & 13 & $-16.7 \pm 0.2$ & $16.0 \pm 0.5$ & $4.8 \pm 0.1$ & 13 & $-16.7 \pm 0.4$ & $15.9 \pm 0.6$ & $4.8 \pm 0.2$ \\
\hline \multicolumn{11}{|l|}{ Beluga } \\
\hline \multirow[t]{2}{*}{ Estuary } & $1988-90$ & $2+$ individuals & 11 & $-16.7 \pm 0.2$ & $15.8 \pm 0.6$ & $4.6 \pm 0.2$ & 16 & $-17.3 \pm 0.2$ & $15.1 \pm 0.4$ & $4.4 \pm 0.1$ \\
\hline & 1997 & $2+$ individuals & 4 & $-16.2 \pm 0.3$ & $16.3 \pm 1.1$ & $4.8 \pm$ & 6 & $-16.7 \pm 0.2$ & $15.3 \pm$ & $4.5 \pm 0.1$ \\
\hline \multicolumn{11}{|l|}{ Grey seal } \\
\hline \multirow[t]{2}{*}{ Estuary } & $1995-96$ & Yearling & 2 & $-18.1 \pm 0.2$ & $14.8 \pm 0.2$ & $4.5 \pm 0.1$ & 8 & $-17.8 \pm 0.4$ & $15.5 \pm 0.5$ & $4.7 \pm 0.2$ \\
\hline & & $2+$ individuals & 5 & $-17.6 \pm 0.6$ & $15.6 \pm 0.9$ & $4.8 \pm$ & 4 & $-17.8 \pm 0.5$ & $15.2 \pm 0.6$ & $4.6 \pm 0.2$ \\
\hline Gulf & 1996 & $2+$ individuals & 8 & $-17.4 \pm 0.5$ & $15.6 \pm 0.7$ & $4.7 \pm 0.2$ & 8 & $-17.7 \pm 0.8$ & $15.0 \pm 0.9$ & $4.6 \pm 0.3$ \\
\hline \multicolumn{11}{|l|}{ Harp seal } \\
\hline \multirow[t]{2}{*}{ Estuary } & 1996 & Yearling & 5 & $-18.3 \pm 0.3$ & $13.1 \pm 0.3$ & $3.8 \pm 0.1$ & 4 & $-18.4 \pm 0.2$ & $13.6 \pm 1.1$ & $4.0 \pm 0.3$ \\
\hline & & $2+$ individuals & 2 & $-17.6 \pm 0.1$ & $13.5 \pm 0.2$ & $3.9 \pm$ & 7 & $-17.6 \pm 0.3$ & $13.7 \pm 0.7$ & $4.0 \pm 0.2$ \\
\hline \multirow[t]{2}{*}{ Gulf } & 1995 & $2+$ individuals & 6 & $-17.7 \pm 0.4$ & $15.5 \pm 0.4$ & $4.7 \pm 0.1$ & 18 & $-17.7 \pm 0.4$ & $14.9 \pm 0.4$ & $4.5 \pm 0.1$ \\
\hline & 1996 & $2+$ individuals & 7 & $-17.9 \pm 0.5$ & $15.0 \pm 0.5$ & $4.6 \pm 0.1$ & 8 & $-17.8 \pm 0.4$ & $14.5 \pm 0.7$ & $4.4 \pm 0.2$ \\
\hline \multicolumn{11}{|l|}{ Hooded seal } \\
\hline \multirow{2}{*}{ Gulf } & 1995 & $2+$ individuals & 7 & $-16.9 \pm 0.4$ & $16.5 \pm 0.7$ & $5.0 \pm 0.2$ & 20 & $-17.0 \pm 0.3$ & $15.7 \pm 0.5$ & $4.8 \pm 0.2$ \\
\hline & 1996 & $2+$ individuals & 9 & $-17.1 \pm 0.3$ & $15.5 \pm 0.6$ & $4.7 \pm 0.2$ & 8 & $-17.3 \pm 0.3$ & $15.8 \pm 0.5$ & $4.8 \pm 0.1$ \\
\hline
\end{tabular}
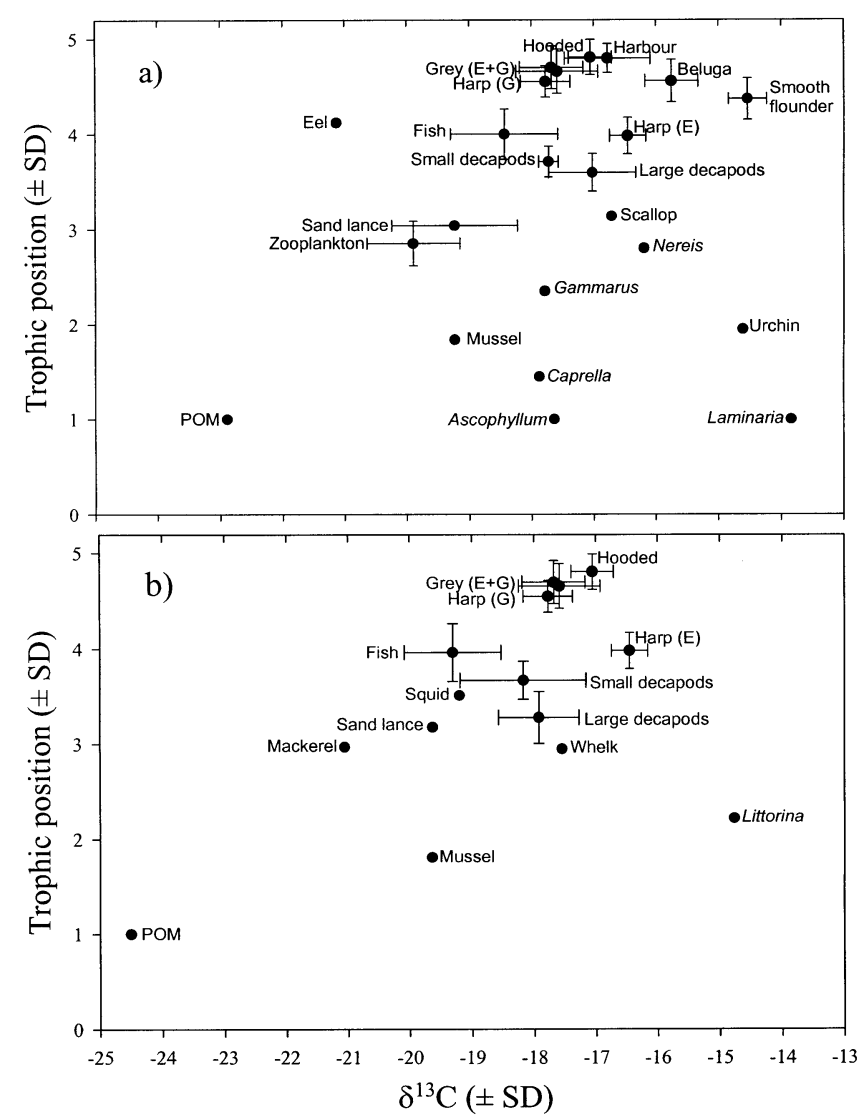

Fig. 4. Trophic structure of (a) Estuary and (b) Gulf of St Lawrence as determined from trophic position and carbon isotope ratios of their different components. $\delta^{13} \mathrm{C}$ value for Gulf POM was obtained from Tan \& Strain (1979). Specific names as in Tables $3 \& 4$ increased with ascending TP from POM through zooplankton, fishes and marine mammals in both regions. A similar increase in ${ }^{13} \mathrm{C}$ abundance with increasing TP was also observed from Ascophyllum nodosum to amphipods, sea urchins, molluscs, and worms to decapods and smooth flounders ${ }^{1}$. Among macroalgae species, ${ }^{13} \mathrm{C}$ contents varied by nearly $4 \%$, with the infralittoral species Laminaria longicruris being enriched compared to the intertidal species Ascophyllum nodosum.

Nitrogen isotope ratios varied by $12 \%$ among organisms from a given region (Fig. 4, Tables $3 \& 4$ ). The least enriched species were primary producers, with $\delta^{15} \mathrm{~N}$ values of 4.5 to $7.9 \%$. Benthic and planktonic invertebrates were enriched in ${ }^{15} \mathrm{~N}$ relative to primary producers, but generally were less enriched than most fishes and marine mammals. Marine mammals were the most enriched group in both the Estuary and Gulf communities, and occupied the highest TP, along with a few fish species such as white hakes and smooth flounders $\geq 32 \mathrm{~cm}$ in length, sea ravens and fourline snakeblennies ${ }^{1}$.

The various species, age class and sex groupings of marine mammals differed significantly in both TP and $\delta^{13} \mathrm{C}$ of their average diet (ANOVA on ranks: $F_{13,236}=$ 21.40 and $F_{15,234}=48.33, \mathrm{p}<0.0001$ for both TP and $\delta^{13} \mathrm{C}$ of diet) (Tables 5, $6 \& 7$ ). Estuary harp seals and female beluga whales occupied the lowest TP of all marine mammals, whereas harbour and hooded seals

\footnotetext{
${ }^{1}$ Corresponding scientific names are presented in Tables $3 \& 4$
} 

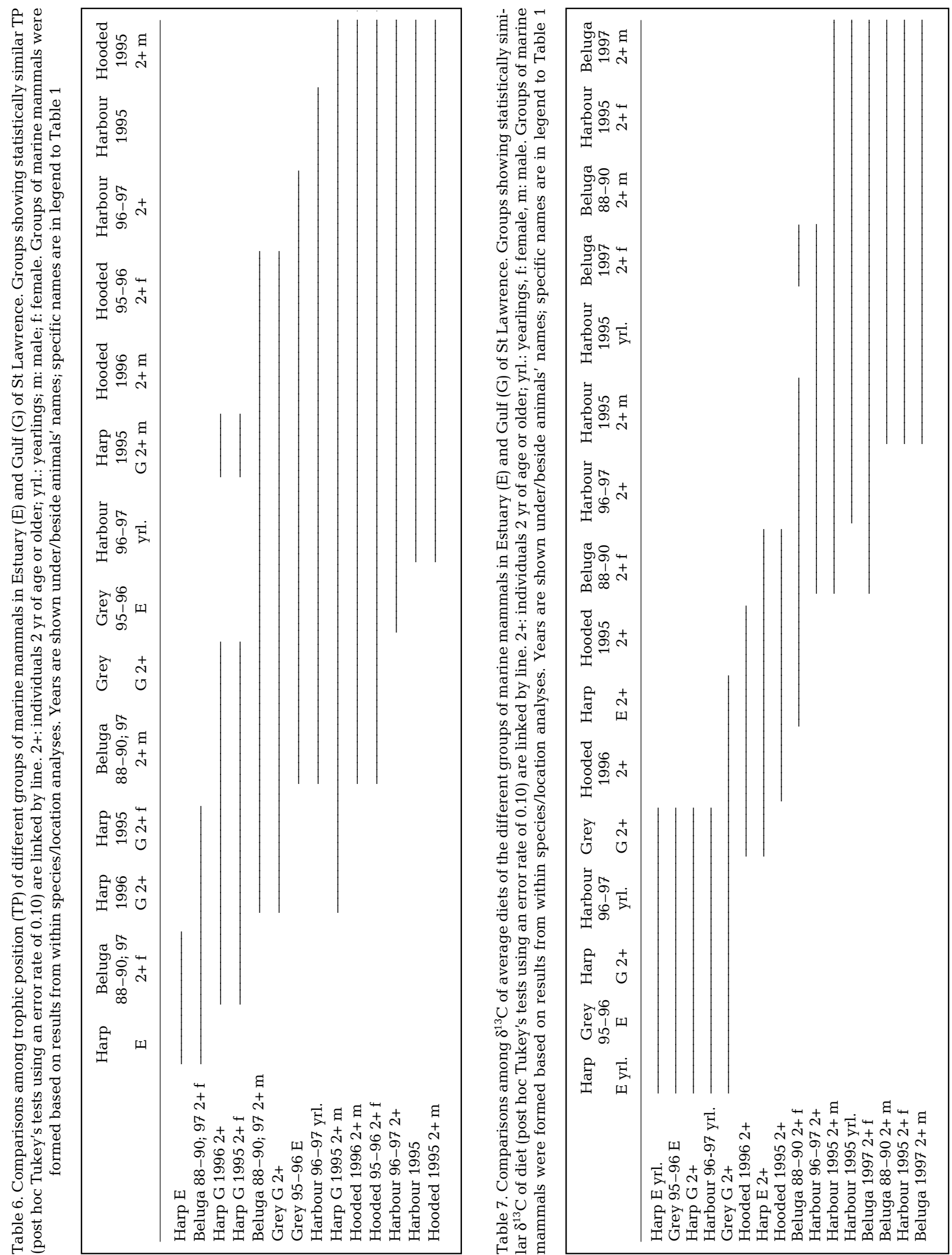
were at the other extreme. Gulf harp seals, grey seals and male beluga whales occupied intermediate TPs. When examining $\delta^{13} \mathrm{C}$ values of the average diet, marine mammal groups segregated further. Harbour seals and beluga whales fed on the most ${ }^{13} \mathrm{C}$-enriched diets, whereas harp seal yearlings from the Estuary, $2+$ harp seals from the Gulf, and grey seals captured either in the Estuary or Gulf fed on the least enriched prey. The only exception was observed in 1996 to 1997 yearling harbour seals, whose diet was similar in $\delta^{13} \mathrm{C}$ to that of grey seals and Gulf harp seals. Diets of Gulf hooded seals and 2+ harp seals from the Estuary were intermediately enriched in ${ }^{13} \mathrm{C}$.

The trophic position and $\delta^{13} \mathrm{C}$ of the average diet also varied over time in Estuary grey and harbour seals. Diets integrated in red blood cells were ${ }^{13} \mathrm{C}$-depleted relative to those integrated in serum in all males and females of both yearling and 2+ grey seals (averages of 0.8 to $1.2 \%$; range 0.3 to $1.5 \%$; paired $t$-tests: $p<0.001$ for each of the age classes) (Fig. 5a). Trophic positions estimated from either tissue were similar for yearling males and females, and for 2+ males and females. In harbour seals captured in late summer, TP and diet integrated by the 2 tissues were similar (Fig. 5b), regardless of age class or sex (all $p>0.05$ ). However, for harbour seals captured in early summer (Fig. 5c), yearling females $\left(\mathrm{p}<0.10\right.$ for both TP and $\delta^{13} \mathrm{C}$ of diet; $\mathrm{n}=3$ ) and $2+$ females ( $<<0.05$ for both TP and $\delta^{13} \mathrm{C}$ of diet; $\mathrm{n}=8)$ had on average a lower TP $(0.1 \%$ for each age class), and fed on diets depleted in ${ }^{13} \mathrm{C}$ by 0.5 and $0.6 \%$ respectively, when diet was estimated from their blood serum rather than from their blood cells. Diets reflected by blood serum or red cells of $2+$ and yearling males captured in early summer were similar in $\delta^{13} \mathrm{C}$ values. However, TPs of $2+$ males were, as in $2+$ females, $0.1 \%$ higher according to blood cells than to serum $(p=0.043 ; n=9)$. When comparing $\delta^{13} \mathrm{C}$ of diet and TP integrated in blood cells to those in hair, males and females of both yearling and 2+ harbour seals occupied similar TPs (all $p>0.10$ ), but were on average 0.8 to $1.1 \%$ o depleted in ${ }^{13} \mathrm{C}$ in their hair relative to their blood cells (range from $0.4 \%$ enriched to $2.1 \%$ depleted; all $\mathrm{p}<0.002$ ).

\section{DISCUSSION}

\section{Patterns of ${ }^{13} \mathrm{C}$ signatures and trophic relationships}

Primary sources differed widely in their $\delta^{13} \mathrm{C}$ values, with POM being, as expected, ${ }^{13} \mathrm{C}$-depleted relative to benthic macroalgae (Fry \& Sherr 1984). Benthic microalgae may represent an important $C$ source for several benthic organisms (Deegan \& Garritt 1997), but benthic microalgae were not sampled in this study. In
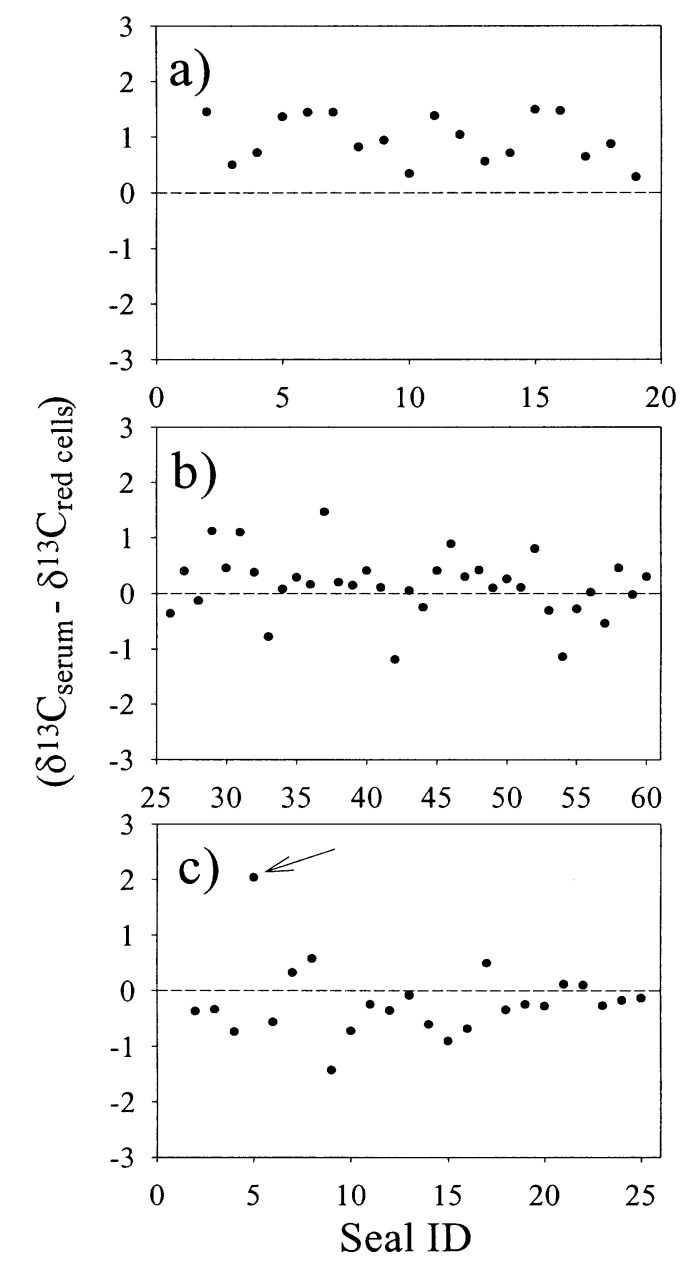

Fig. 5. Differences in $\delta^{13} \mathrm{C}$ values between blood serum and red cells of (a) Estuary-captured grey seals, Halichoerus grypus, (b) harbour seals Phoca vitulina captured during or later than July and (c) harbour seals captured before July. Values above zero line indicate seals that have red blood cells depleted in ${ }^{13} \mathrm{C}$ relative to blood serum. Values located well above zero line (many individuals in [a] and individual indicated by arrow in [c]) indicate animals that might have recently visited Gulf of St Lawrence

other estuarine systems, benthic microalgae had $\delta^{13} \mathrm{C}$ values that were similar to macroalgae and $\delta^{15} \mathrm{~N}$ values that were similar to POM (Riera et al. 1996, Page 1997). Macroalgae from the infralittoral (Laminaria longicruris) and intertidal (Ascophyllum nodosum) zones differed markedly in their $\delta^{13} \mathrm{C}$ values. This large variation $(4 \%)$ is likely attributable to lower turbulence and recycling of dissolved $\mathrm{C}$ in the boundary layer of infralittoral species, resulting in a progressive increase in ${ }^{13} \mathrm{C}$ concentration, as well as greater access by intertidal species to ${ }^{13} \mathrm{C}$-depleted air $\mathrm{CO}_{2}$ during emersion (Osmond et al. 1981, Surif \& Raven 1990). Despite this variation, macroalgae still differed from POM by 5.3 to $9.1 \%$. 
Our findings on community structure of the Estuary and Gulf of St Lawrence are limited by small sample sizes of several community components. However, $2 \mathrm{C}$ pathways or components were observed in the trophic food webs. One involved POM and pelagic-dependent species, while the other involved benthic micro- or macroalgae and more benthic species (Fig. 4). The variation in $\delta^{13} \mathrm{C}$ within each component suggests that some mixing between the 2 pathways might occur. This could result from sedimentation of phytoplankton, consumers feeding in both benthic and pelagic regions, or from re-suspension of benthic algae and marsh detritus. Most fishes were ${ }^{13} \mathrm{C}$-enriched relative to POM and zooplankton, but depleted relative to benthic algae, indicating that the pelagic component of the system was where they accrued most of their food resources. Species such as Littorina sp., sea urchins, Gammarus sp., Nereis spp. worms, blue mussels, decapods, smooth flounders, Atlantic tomcod and Cottidae ${ }^{1}$ were 1.0 to $5.1 \%$ enriched in ${ }^{13} \mathrm{C}$ relative to pelagicfeeding species from a similar TP such as herring, capelin or redfish ${ }^{1}$, and likely depend to a greater extent on benthic resources. Marine mammals were generally ${ }^{13} \mathrm{C}$-enriched relative to $\mathrm{POM}$ and slightly depleted relative to benthic macroalgae, suggesting that none of them relied exclusively on benthic resources. However, some benthic or demersal organisms are known to be important prey for some of these species, e.g. Greenland halibut ${ }^{1}$ for hooded seals (Ross 1993), and Nereis spp. worms and shrimps for beluga whales (Vladykov 1946). These probably contribute to increasing the $\delta^{13} \mathrm{C}$ values of these marine mammals relative to those of species that depend heavily on pelagic prey.

Factors such as distribution may also have contributed to the $\delta^{13} \mathrm{C}$ variation observed among and within the marine mammal species studied. $\delta^{13} \mathrm{C}$ of a $\mathrm{C}$ source may differ among regions (Dunton et al. 1989, this study), resulting in consumers having different $\delta^{13} \mathrm{C}$ values despite the exploitation of the same resources. The Lower Estuary POM is generally enriched in ${ }^{13} \mathrm{C}$ by 2 to $3.5 \%$ compared to the Upper Estuary or Gulf POM (ca $-25 \%$ ). This is probably the result of a high $\mathrm{C}$ demand or a change in the phytoplanktonic species that dominate these areas (Tan \& Strain 1979, 1983). The ${ }^{13} \mathrm{C}$-depletion observed in Gulf harp and grey seals relative to Estuary harbour seals and beluga whales may result from an exploitation of ${ }^{13} \mathrm{C}$-depleted Gulf resources by these species. The similarity of $\delta^{13} \mathrm{C}$ values between Estuary grey seals and seals from the Gulf and the differences between the short-term (serum) and long-term (cells) diet integrators indicate

\footnotetext{
${ }^{1}$ Corresponding scientific names are presented in Tables $3 \& 4$
}

that their tissues still reflect the isotopic signature of resources exploited while in the Gulf (Fig. 5a). Therefore, it is likely that grey seals captured in the Lower Estuary had only recently entered the area. These data concur with anecdotal observations that grey seals over-winter outside the Estuary and return to this region in the late spring (Lavigueur et al. 1993).

Similarly, the ${ }^{13} \mathrm{C}$-depletion of harp seals sampled in March on the whelping patch in the Gulf suggests that few of them had visited the Estuary. However, harp seals captured in February in the Estuary were more similar to Estuary harbour seals or beluga whales than to other species from the Gulf when their lower trophic position was accounted for, i.e. when $\delta^{13} \mathrm{C}$ values were corrected using a $1.3 \%{ }^{13} \mathrm{C}$-enrichment per trophic level (Table 2). The $\delta^{13} \mathrm{C}$ value of Estuary harp seals suggests that they had been in the Estuary since at least early December, assuming a half-life of 4 wk for muscle tissue and a spread over 2 to $3 \mathrm{mo}$ in $\delta^{13} \mathrm{C}$ changes (Tieszen et al. 1983). This coincides with the seasonal timing of observations of large numbers of harp seals in the Lower Estuary (Sergeant 1991).

Gulf hooded seals had $\delta^{13} \mathrm{C}$ values that were intermediate between those of Estuary and Gulf animals, suggesting that they might have visited the Estuary in the months preceding their capture. Alternatively, their higher enrichment relative to other Gulf species might have resulted from a greater dependence on benthic or demersal resources. Both hypotheses are possible. Although the proportion of hooded seals entering the Estuary each year is largely unknown, a greater number of hooded seal winter sightings were made in 1996 in the Estuary compared to other years (E. Albert \& L. Measures, Fisheries and Oceans Canada, Mont-Joli, Quebec, unpubl. data). Northwest Atlantic hooded seals are also reported to ingest large quantities of demersal Greenland halibut, principally in the size range from 20 to $40 \mathrm{~cm}$ (Hammill et al. 1997). The $\delta^{13} \mathrm{C}$ of their average diet was about $-18.8 \%$ and was, therefore, compatible with Greenland halibut of this size class from either the Estuary $(-18.6$ to $-19.2 \%$ ) or Gulf $(-18.7$ to $-19.8 \%$ ) (Tables $3 \& 4)$.

Male beluga whales were significantly enriched in ${ }^{13} \mathrm{C}$ relative to females, suggesting either a larger dependence by males on benthic species or different distributions of the 2 sexes. It is unlikely that the larger ${ }^{13} \mathrm{C}$-enrichment of males is related to more intense benthic feeding, since the observations from stable isotopes are opposite to what would be expected from the limited diet data available for this species in the $\mathrm{St}$ Lawrence Estuary (Vladykov 1946). The lower $\delta^{15} \mathrm{~N}$ values of female beluga whales suggests that females feed at lower trophic levels than males, and therefore, that their ${ }^{13} \mathrm{C}$-depletion relative to males might in part result from their lower trophic position. However, some 
of this variability might also result from seasonal segregation between the sexes. During the summer, adult females are observed most frequently in the ${ }^{13} \mathrm{C}$ depleted Upper Estuary, and adult males mainly in the ${ }^{13}$ C-enriched Lower Estuary (Kingsley 1993, Michaud 1993).

The comparison of carbon isotope ratios in short- and longer-term integrators of diet provided little evidence for seasonal movements of harbour seals outside the Estuary. If significant excursions into the Upper Estuary or Gulf of St Lawrence had occurred during winter, $\delta^{13} \mathrm{C}$ of short-term integrators should have been ${ }^{13} \mathrm{C}$ enriched (Lower Estuary) relative to longer-term integrators. Although these results must be interpreted with caution given that seasonal shifts in diet are possible, the data obtained in this study are in agreement with the limited harbour seal satellite-telemetry data available for this area (Lesage 1999), and support the hypothesis that harbour seals are relatively sedentary (Boulva \& McLaren 1979, Thompson 1993).

Although it appears that most of the variation observed in $\delta^{13} \mathrm{C}$ may be explained by species' distributions, some variation among sympatric species was probably related to food preferences. The slightly higher $\delta^{13} \mathrm{C}$ values observed in most beluga whales relative to harbour seals suggests a greater reliance by beluga whales on benthic or demersal prey. The ingestion of a larger proportion of invertebrates by beluga whales would also be consistent with their lower TP relative to harbour seals. Contents from 2 stomachs collected in 1989 and 1995, and diet reports from the late 1930s, indicate that invertebrates such as Nereis spp. worms and shrimp-like decapods, as well as Atlantic tomcod (150 to $200 \mathrm{~mm}$ ), sand lance (140 to $185 \mathrm{~mm}$ ) and redfish may be important species in the St Lawrence beluga's diet, along with the more pelagic capelin (135 to $180 \mathrm{~mm}$ ) (Vladykov 1946, Béland 1995, Lesage \& Hammill unpubl. data). Sand lance and capelin are commonly found in the stomach of St Lawrence harbour seals, while shrimp-like decapods or other benthic invertebrates occur only occasionally (Lesage, Hammill \& Kovacs unpubl. data). The higher $\delta^{13} \mathrm{C}$ in hooded seals relative to other Gulf species may result from their preference for more ${ }^{13} \mathrm{C}$-enriched demersal prey, e.g. Greenland halibut (Ross 1993, Hammill et al. 1997). According to their $\delta^{13} \mathrm{C}$ values, Greenland halibut (26 to $36 \mathrm{~cm}$ ) and Arctic cod (13 to $21 \mathrm{~cm})$, the 2 most important prey of hooded seals off Newfoundland during winter (Ross 1993), would account for 71 and $29 \%$ of the seals' diet. However, $\delta^{15} \mathrm{~N}$ values of both prey (ca 15.3\%) are very close to that of hooded seals (ca 15.7\%o for all classes of hooded seals except 1995 males), which suggests that hooded seals in the Gulf also fed at lower trophic levels or they fed predominantly on smaller $(<20 \mathrm{~cm})$ Greenland halibut than the 26 to $36 \mathrm{~cm}$ range reported by Ross (1993) (Table 4).

\section{Patterns of ${ }^{15} \mathrm{~N}$ signatures and trophic relationships}

The St Lawrence Estuary and Gulf systems each consisted of at least 5 trophic levels (Fig. 4). Killer whales, whose diet may include seals and whales, would probably add a further trophic level to the Gulf system. Most ecosystems appear to be limited to 4-6 trophic levels, with terrestrial chains generally being shorter (Briand \& Cohen 1987, Schoener 1989). Factors constraining food chain length are controversial. Food chains may remain short due to the progressive depletion of energy transferred to higher trophic levels, or because long food chains may suffer population fluctuations so severe as to impair the persistence of top predators (reviewed in Pimm 1982, DeAngelis 1995, Power et al. 1996). Recent hypotheses that have not yet been fully explored involve dimensionality of habitats, i.e. 3-dimensional habitats may support longer chains than 2-dimensional habitats (Briand \& Cohen 1987, Pimm et al. 1991) and species richness, i.e. chains should be longer in communities supporting a larger number of species (Bengtsson 1994, Martinez \& Lawton 1995).

Nitrogen isotopes appeared to fractionate similarly in Estuary and Gulf POM (Gearing \& Pocklington 1990) and more consistently among different taxonomic groups than $\mathrm{C}$ isotopes (reviewed in Lesage 1999) and are, therefore, a better marker of TP. Zooplankton species did not appear to rely directly on phytoplankton, since they occupied TPs of 2.5 to 3.0. In aquatic systems in particular, predator-prey relationships are often determined largely by size (Werner \& Gilliam 1984). Phytoplankton in the Lower Estuary is dominated by small-sized diatoms $(<25 \mu \mathrm{m}$; Levasseur et al. 1984) and zooplankton collected in this study were generally $>160 \mu \mathrm{m}$. Planktonic ciliated protozoa (25 to $75 \mu \mathrm{m})$, intermediate in size between phytoplankton and the sampled mesozooplankton, are regarded as an important trophic link between these 2 groups in the Lower Estuary (Sime-Ngando et al. 1995). Similarly, the dominant copepod in the Gulf, Calanus finmarchicus, may rely mainly on heterotrophic microzooplankton for egg production and net lipid synthesis (Ohman \& Runge 1994). The absence of species at TP 2 in this study and the 1.7 to $4.0 \%$ ${ }^{13} \mathrm{C}$-enrichment of mesozooplankton relative to POM (Table 3), i.e. nearly twice the 1.3 to $2.3 \%$ trophic enrichments reported for lipid-free tissues of marine organisms (reviewed in Lesage 1999), support the hypothesis that protozoa are the main primary consumers of POM in the Estuary. Nevertheless, some 
macrozooplankton such as C. hyperboreus, which showed relatively low TP and $\delta^{15} \mathrm{~N}$, probably had mixed diets of microzoo- and phytoplankton. Larger decapods varied widely in $\mathrm{TP}$, perhaps because of their habit of feeding on a variety of benthic invertebrates including grazers (e.g. sea urchins), consumers of necrotic molluscs (e.g. whelks) and suspension feeders (e.g. some bivalves). Trophic positions varied little amongst most fishes, which probably preyed to a large extent on macrozooplankton. However, decapods, smaller fishes or fish larvae probably became prominent in the diet of larger and more ${ }^{15} \mathrm{~N}$-enriched fishes such as white hakes, sea ravens, large Atlantic cod, redfish and some Cottidae.

Marine mammals occupied the top positions in both webs and, according to the narrow range of their TP, overlapped trophically with each other. However, an overlap in TP does not necessarily imply an overlap in diet, since animals may feed on different species but still occupy similar TPs if the various prey that they eat result in a similar TP. The extent of the trophic or diet overlap between species was difficult to ascertain in this study, because diet was not integrated over exactly the same period among the different marine mammals. Harbour seals and beluga whales that occupy the Estuary throughout the year could only be sampled during the summer, whereas harp seals and hooded seals that normally summer in the Arctic were only sampled during winter. Grey seals captured in summer in the Estuary had signatures reflecting a Gulf diet, and there is some uncertainty as to whether harp, grey and hooded seals sampled in the Gulf actually entered the Estuary prior to sampling. Nevertheless, it is unlikely that 2 species overlapped completely in their resource requirements (Schoener 1974). Often, potential competitors will either occupy different habitats so that they do not really coexist on a local scale, or will differ in body size, resulting in slight differences in diet composition or preferred prey size. A 2 -fold difference in mass, or 1.25 ratio if using linear dimensions, is often considered sufficient for competition avoidance (Schoener 1974, Bowers \& Brown 1982). Among the marine mammals in this study, important variations in body size occur between and within species. The $2+$ animals are larger than yearlings and 2+ males are larger than 2+ females. Harp and harbour seals are relatively similar in body mass ( 110 to 130 and 80 to $120 \mathrm{~kg}$, respectively), but are considerably smaller than grey and hooded seals ( 200 to 350 and 260 to $400 \mathrm{~kg}$, respectively) (Reeves et al. 1992). Adult beluga whales are much larger than any other species, with a mean mass of 700 to $970 \mathrm{~kg}$ (this study).

The similarly-sized harp and harbour seals differed most in their TPs, while harbour versus hooded seals, and grey versus Gulf harp seals were the species which overlapped most closely trophically. However, these species appeared to differ in their habitat requirements, which reduces the possibility of actual resource overlap. Harbour seals remain in the Estuary and probably feed, to a large extent, on pelagic species found near the coast, whereas hooded seals probably remain in the Gulf and feed mostly on offshore demersal species. Grey seals are relatively coastal (Thompson et al. 1991, J.-F. Gosselin, Fisheries and Oceans Canada, Mont-Joli, Quebec, unpubl. data), whereas harp seals are mainly pelagic (Sergeant 1991). Estuary harp seals and beluga whales overlapped in their TPs, but their average diet differed significantly in $\delta^{13} \mathrm{C}$, indicating the use of different resources. Indeed, the stomachs of harp seals sampled in this study contained mainly capelin and euphausiids, although sand lance and crustaceans were also regularly consumed (Hammill unpubl. data). Assuming that only capelin and euphausiids were ingested, the latter would account for up to $75 \%$ of the Estuary harp seal diet. The greater reliance of beluga whales on ${ }^{13} \mathrm{C}$-enriched benthic or demersal invertebrates and of harp seals on ${ }^{13} \mathrm{C}$ depleted euphausiids could explain the divergence in their $\delta^{13} \mathrm{C}$ values and their similarity in TP.

An overlap in food preferences may also have been possible between $2+$ animals of small species and yearlings of larger species. Juvenile grey seals and adult harbour seals, 2 similarly sized groups, often hauled out on rocks very close to each other. However, slight differences were observed in the TP of adult harbour seals relative to Estuary grey seals, which consisted mainly of subadult or yearling individuals, indicating some partitioning of food resources. Satellite telemetry data from adult harbour seals and subadult grey seals indicated that subadult grey seals consistently dove to deeper depths than the harbour seals while at sea (Lesage et al. 1995), suggesting spatial partitioning of foraging areas in a 3-dimensional sense.

Differences in body size, diving capabilities or food handling ability between age classes (Scholander et al. 1942, Kleiber 1961) may result in animals feeding on different trophic levels as they mature (life-history omnivory; Werner \& Gilliam 1984, Sprules \& Bowerman 1988). Many species of fishes become more piscivorous with age, which would increase their trophic position relative to juvenile conspecifics. In marine mammals, lower prey capture or handling skills and diving ability in young individuals (Le Boeuf et al. 1996, Horning \& Trillmich 1997, Lesage 1999) would promote feeding at lower trophic levels (e.g. Boulva \& McLaren 1979, Lawson et al. 1995, Proust 1995) and would reduce resource overlap with older animals. Isotopic $\mathrm{C}$ and $\mathrm{N}$ provided evidence of life-history omnivory in both marine mammals and fishes. In species where this phenomenon was observed, smaller 
individuals were ${ }^{13} \mathrm{C}$-depleted or ${ }^{15} \mathrm{~N}$-depleted relative to larger individuals, suggesting that they fed at lower trophic levels. Life-history omnivory has been documented from stomach content examination (e.g. Werner \& Gilliam 1984, Huse \& Toresen 1996), and from stable isotope analysis (e.g. Gearing et al. 1984, Hobson \& Welch 1995, France 1996). The $\delta^{15} \mathrm{~N}$ values of yearling harp seals from the Estuary indicated that they feed at slightly lower trophic levels than older individuals from the same region. Stomach contents from both yearling and older Estuary harp seals contained capelin, sand lance, euphausiids and small crustaceans. The yearlings' diet consisted primarily of euphausiids and a few capelin, whereas older individuals consumed a combination of capelin and euphausiids (Hammill unpubl. data). Conclusions drawn from mixing models were not as dramatic, although they should be interpreted with caution. In model simulations that assumed only capelin and euphausiids were ingested, capelin represented a slightly lower proportion of the yearlings' diet $(23 \%)$ compared to that of older individuals $(32 \%)$. The lower TP of Estuary harp seals compared to those captured in the Gulf may in part be an age-related phenomenon, since Estuary harp seals were mostly sexually immature, whereas all Gulf harp seals were sexually mature.

Very little information exists on the diet of yearlings and 2+ St Lawrence harbour seals. Capelin and sand lance were the 2 most common prey found in the few non-empty stomach contents taken from weaned pups ( $\mathrm{n}=8$ ), with invertebrate parts also being observed occasionally (Lesage, Hammill \& Kovacs unpubl. data). These prey were also observed frequently in the diet of yearlings $(n=5)$ and older individuals $(n=3)$, along with herring (both yearlings and older individuals) and a few Cottidae and Pleuronectidae. The relative importance of capelin and herring in the harbour seal diet cannot be determined because their $\mathrm{C}$ and $\mathrm{N}$ isotopic values are similar. However, their contribution to the diet relative to that of sand lance may be obtained through mixing models, given the $2 \%$ difference between the 2 groups. Sand lance would have contributed up to $70 \%$ of the diet of yearlings, but less than $26 \%$ of that of older individuals.

Body size dimorphism between males and females may also have led to differences in TP. Adult males of the dimorphic species (beluga whales, hooded and grey seals, and to a much lesser extent harp seals) generally occupied higher TPs than females, and may have consumed food resources inaccessible to or too large for females (Kooyman 1989, Boyd \& Croxall 1996). Sex-related variation in TP was not observed in Estuary harbour seals, and may have been the result of a less pronounced physical dimorphism in this species. Differences in TP between sexes were also not ob- served in 2+ Estuary grey and harp seals, probably as a result of the relatively similar size of the 2 sexes in these immature animals.

The use of multiple tissues and multiple stable isotopes has provided insights into the trophic relationships of both the Estuary and Gulf of St Lawrence ecosystems. This approach had highlighted 2 major carbon pathways or components in these trophic webs, in addition to providing a valuable framework or validation tool for the construction of multispecies ecosystem models. Marine mammals such as seals and beluga whales occupy top trophic positions in both of these ecosystems, and appear to be mainly piscivorous. Although some resource overlap appears to occur with other marine mammals, it is likely that the combination of seasonal changes in distribution and habitat would reduce resource overlap and, consequently, the potential for competition. More complete sampling is required to examine the question further. The stable isotope approach is limited in its ability to identify the contribution of individual species occupying the same trophic level. However, this approach provides a reliable picture of the contribution of invertebrates to the diet and indicates that some species (harp seals and beluga whales) may derive a considerable amount of their energy from invertebrate food resources.

Acknowledgements. Special thanks are extended to W. D. Bowen, R. Doucett, J. O'Hara Hines, G. Power and W. Taylor for commenting on the manuscript; K. E. Bernt, M. Deakos, J.-F. Gosselin, C. Mursell, S. E. Picard, and I. Welch helped with the fieldwork; P. Carter, A. Evely, J.-F. Gosselin and F. Proust helped with sample preparation, age determination and stomach content identification. We are also grateful to the Institut National d'Écotoxicologie du Saint-Laurent, whose personnel collected beluga samples from 1988-1992, and to D. Archambault, G. Chouinard, S. Bérubé, P. Joly, R. Morneau, and B. Sainte-Marie for providing prey samples. We thank R. Drimmie, M. E. Patton and W. Mark from the Environmental Isotope Laboratory (Waterloo, Ontario) for analysing the samples, and $\mathrm{K}$. A. Hobson for his advice in the early stages of the study. Financial support was provided to V.L. by the Fonds pour la Formation de Chercheurs et l'Aide à la Recherche (FCAR), Canadian Wildlife Foundation, University of Waterloo Graduate Scholarship Program, and the Davis Memorial Fund. This research project was funded under the St Lawrence Action Plan-Vision 2000 research program of the Canadian Department of Fisheries and Oceans and the Green Plan of Parks Canada.

\section{LITERATURE CITED}

Alexander SA, Hobson KA, Gratto-Trevor CL, Diamond AW (1996) Conventional and isotopic determinations of shorebird diets at an inland stopover: the importance of invertebrates and Potamogeton pectinatus tubers. Can J Zool 74: $1057-1068$

Béland P (1995) Mortalités de bélugas observées dans le Saint-Laurent en 1995. INESL, Rimouski, QC, for the Department of Fisheries and Oceans, Mont-Joli, QC 
Ben-David M, Flynn RW, Schell DM (1997) Annual and seasonal changes in diets of martens: evidence from stable isotope analysis. Oecologia 111:280-291

Bengtsson J (1994) Confounding variables and independent observations in comparative analyses of food webs. Ecology 75:1282-1288

Bernt KE, Hammill MO, Kovacs KM (1996) Age estimation of grey seals (Halichoerus grypus) using incisors. Mar Mamm Sci 12:476-482

Bligh EG, Dyer WJ (1959) A rapid method of total lipid extraction and purification. Can J Biochem Physiol 37:911-917

Boulva J, McLaren IA (1979) Biology of the harbor seals, Phoca vitulina, in eastern Canada. Bull Fish Res Board Can 200:1-24

Bowen WD (1997) Role of marine mammals in aquatic ecosystems. Mar Ecol Prog Ser 158:267-274

Bowers MA, Brown JH (1982) Body size and coexistence in desert rodents: chance or community structure? Ecology 63:391-400

Boyd IL, Croxall JP (1996) Dive durations in pinnipeds and seabirds. Can J Zool 74:1696-1705

Briand F, Cohen JE (1987) Environmental correlates of food chain length. Science 238:956-960

Brodie PF, Geraci JR, St Aubin DJ (1990) Dynamics of tooth growth in beluga whales, Delphinapterus leucas, and effectiveness of tetracycline as a marker for age determination. In: Smith TG, St Aubin DJ, Geraci JR (eds) Advances in research on the beluga whale, Delphinapterus leucas. Can Bull Fish Aquat Sci 224:141-148

Bunn SE, Loneragan NR, Kempster MA (1995) Effects of acid washing on stable isotope ratios of $\mathrm{C}$ and $\mathrm{N}$ in penaeid shrimp and seagrass: implications for food-web studies using multiple stable isotopes. Limnol Oceanogr 40:622-625

Conover WJ (1980) Practical nonparametric statistics, 2nd edn. John Wiley \& Sons, New York

DeAngelis DL (1995) Relationships between the energetics of species and large-scale species richness. In: Jones CG, Lawton JH (eds) Linking species and ecosystems. Chapman \& Hall, New York, p 263-272

Deegan LA, Garritt RH (1997) Evidence for spatial variability in estuarine food webs. Mar Ecol Prog Ser 147:31-47

Dunton KH, Saupe SM, Golikov AN, Schell DM, Schonberg SV (1989) Trophic relationships and isotopic gradients among arctic and subarctic marine fauna. Mar Ecol Prog Ser 56:89-97

El-Sabh MI, Silverberg N (1990) Oceanography of a largescale estuarine system. The St Lawrence. Springer-Verlag, Berlin

France RL (1995) Carbon-13 enrichment in benthic compared to planktonic algae: food web implications. Mar Ecol Prog Ser 124:307-312

France RL (1996) Ontogenetic shift in crayfish $\delta^{13} \mathrm{C}$ as a measure of land-water ecotonal coupling. Oecologia 107:239-242

Fry B (1996) ${ }^{13} \mathrm{C} /{ }^{12} \mathrm{C}$ fractionation by marine diatoms. Mar Ecol Prog Ser 134:283-294

Fry B, Sherr EB (1984) $\delta^{13} \mathrm{C}$ measurements as indicators of carbon flow in marine and freshwater ecosystems. Contrib Mar Sci 27:13-47

Gearing JN (1991) The study of diet and trophic relationships through natural abundance ${ }^{13} \mathrm{C}$. In: Coleman DC, Fry B (eds) Carbon isotope techniques. Academic Press, Toronto, p 201-218

Gearing JN, Pocklington R (1990) Organic geochemical studies in the St. Lawrence Estuary. In: El-Sabh MI, Silverberg $\mathrm{N}$ (eds) Oceanography of a large-scale estuarine system: the St. Lawrence. Coastal and estuarine studies, Vol 39. Springer-Verlag, Berlin, p 170-201
Gearing JN, Gearing PJ, Rudnick DT, Requejo AG, Hutchins MJ (1984) Isotopic variability of organic carbon in a phytoplankton-based, temperate estuary. Geochim Cosmochim Acta 48:1089-1098

Hair JF, Anderson RE, Tatham RL, Black WC (1995) Multivariate data analysis: with readings, 4th edn. Prentice Hall, Upper Saddle River, NJ

Hammill MO, Lydersen C, Kovacs KM, Sjare B (1997) Estimated fish consumption by hooded seals (Cystophora cristata) in the Gulf of St. Lawrence. J Northw Atl Fish Sci 22:249-257

Hilderbrand GV, Farley SD, Robbins CT, Hanley TA, Titus K, Servheen C (1996) Use of stable isotopes to determine diets of living and extinct bears. Can J Zool 74:2080-2088

Hobson KA, Clark RG (1992a) Assessing avian diets using stable isotopes, II. Factors influencing diet-tissue fractionation. Condor 94:189-197

Hobson KA, Clark RG (1992b) Assessing avian diets using stable isotopes, I. Turnover of ${ }^{13} \mathrm{C}$ in tissues. Condor 94: 181-188

Hobson KA, Welch HE (1992) Determination of trophic relationships within a high Arctic marine food web using $\delta^{13} \mathrm{C}$ and $\delta^{15} \mathrm{~N}$ analysis. Mar Ecol Prog Ser 84:9-18

Hobson KA, Welch HE (1995) Cannibalism and trophic structure in a high Arctic lake: insights from stable-isotope analysis. Can J Fish Aquat Sci 52:1195-1201

Hobson KA, Schell DM, Renouf D, Noseworthy E (1996) Stable carbon and nitrogen isotopic fractionation between diet and tissues of captive seals: implications for dietary reconstructions involving marine mammals. Can J Fish Aquat Sci 53:528-533

Horning M, Trillmich F (1997) Ontogeny of diving behaviour in the Galápagos fur seal. Behaviour 134:1211-1257

Huse G, Toresen R (1996) A comparative study of the feeding habits of herring (Clupea harengus, Clupeidae, L.) and capelin (Mallotus villosus, Osmeridae, Müller) in the Barents Sea. Sarsia 81:143-153

Kingsley MCS (1993) Census, trend, and status of the St Lawrence beluga population in 1992. Can Tech Rep Fish Aquat Sci 1938:1-17

Kleiber M (1961) The fire of life. Wiley, New York

Kooyman GL (1989) Diverse divers: physiology and behavior. Springer-Verlag, Berlin

Lavigueur L, Hammill MO, Asselin S (1993) Distribution et biologie des phoques et autres mammifères marins dans la région du parc marin du Saguenay. Rapp Manus Can Sci Halieut Aquat 2220:1-40

Lawson JW, Stenson GB, McKinnon DG (1995) Diet of harp seals (Phoca groenlandica) in nearshore waters of the northwest Atlantic during 1990-1993. Can J Zool 73: 1805-1818

Le Boeuf BJ, Morris PA, Blackwell SB, Crocker DE, Costa DP (1996) Diving behavior of juvenile northern elephant seals. Can J Zool 74:1632-1644

Lesage V (1999) Trophic relationships, seasonal diving activity and movements of harbour seals, Phoca vitulina concolor, in the St Lawrence River Estuary, Canada. PhD thesis. University of Waterloo, Waterloo, ON

Lesage V, Hammill MO, Kovacs KM (1995) Comparison of dive behaviour and movements of harbour and grey seals from the St Lawrence Estuary, Quebec, Canada. In: Eleventh Biennial Conference on the Biology of Marine Mammals, 14-18 December, Orlando, FL (Abstract). Society for Marine Mammalogy, San Francisco, CA

Levasseur M, Therriault JC, Legendre L (1984) Hierarchical control of phytoplankton succession by physical factors. Mar Ecol Prog Ser 19:211-222 
Martinez ND, Lawton JH (1995) Scale and food-web structure-from local to global. Oikos 73:148-154

McConnaughey T, McRoy CP (1979) Food-web structure and the fractionation of carbon isotopes in the Bering Sea. Mar Biol 53:257-262

Michaud R (1993) Distribution estivale du béluga du SaintLaurent; synthèse 1986 à 1992. Rapp Tech Can Sci Halieut Aquat 1906:1-28

Minagawa M, Wada E (1984) Stepwise enrichment of ${ }^{15} \mathrm{~N}$ along food chains: further evidence and the relation between $\delta^{15} \mathrm{~N}$ and animal age. Geochim Cosmochim Acta 48:1135-1140

Muelbert MMC, Bowen WD (1993) Duration of lactation and postweaning fast, changes in mass and body composition of harbour seal, Phoca vitulina, pups. Can J Zool 71: 1405-1414

Muir DCG, Segstro MD, Hobson KA, Ford CA, Stewart REA, Olpinski S (1995) Can seal eating explain elevated levels of PCBs and organochlorine pesticides in walrus blubber from eastern Hudson Bay (Canada)? Environ Pollut 96: 335-348

Ohman MD, Runge JA (1994) Sustained fecundity when phytoplankton resources are in short supply: omnivory by Calanus finmarchicus in the Gulf of St. Lawrence. Limnol Oceanogr 39:21-36

Osmond CB, Valaane N, Haslam SM, Uotila P, Roksandic Z (1981) Comparisons of $\delta^{13} \mathrm{C}$ values in leaves of aquatic macrophytes from different habitats in Britain and Finland; some implications for photosynthetic processes in aquatic plants. Oecologia 50:117-124

Ostrom PH, Lien J, Macko SA (1993) Evaluation of the diet of Sowerby's beaked whale, Mesoplodon bidens, based on isotopic comparisons among northwestern Atlantic cetaceans. Can J Zool 71:858-861

Page HM (1997) Importance of vascular plant and algal production to macro-invertebrate consumers in a southern California salt marsh. Estuar Coast Shelf Sci 45:823-834

Pimm SL (1982) Food webs. Chapman \& Hall, London

Pimm SL, Lawton JH, Cohen JE (1991) Food web patterns and their consequences. Nature 350:669-674

Pinnegar JK, Polunin NVC (1999) Differential fractionation of $\delta^{13} \mathrm{C}$ and $\delta^{15} \mathrm{~N}$ among fish tisues: implications for the study of trophic interactions. Funct Ecol 13:225-231

Power ME, Parker MS, Wootton JT (1996) Disturbance and food chain length in rivers. In: Polis GA, Winemiller KO (eds) Food webs: integration of patterns and dynamics. Chapman \& Hall, New York, p 286-297

Proust F (1995) Composition du régime alimentaire du phoque gris (Halichoerus grypus) dans le Golfe du SaintLaurent, Québec, Canada. MSc thesis. Université du Québec à Rimouski, Rimouski, QC

Ramsay MA, Hobson KA (1991) Polar bears make little use of terrestrial food webs: evidence from stable-carbon isotope analysis. Oecologia 86:598-600

Reeves RR, Stewart BS, Leatherwood S (1992) The Sierra Club handbook of seals and sirenians. Sierra Club Book, San Francisco, CA

Riera P, Richard P, Grémare A, Blanchard G (1996) Food

Editorial responsibility: Otto Kinne (Editor),

Oldendorf/Luhe, Germany source of intertidal nematodes in the Bay of MarennesOléron (France), as determined by dual stable isotope analysis. Mar Ecol Prog Ser 142:303-309

Ross SA (1993) Food and feeding of the hooded seal (Cystophora cristata) in Newfoundland. MSc thesis, Memorial University of Newfoundland, St. John

Schoener TW (1974) Resource partitioning in ecological communities. Science 185:27-39

Schoener TW (1989) Food webs from the small to the large. Ecology 70:1559-1589

Scholander PF, Irving L, Grinnell SW (1942) On the temperature and metabolism of the seal during diving. J Cell Comp Physiol 19:67-78

Sergeant DE (1991) Harp seals, man and ice. Can Spec Publ Fish Aquat Sci 114:1-153

Sime-Ngando T, Gosselin M, Roy S, Chanut JP (1995) Significance of planktonic ciliated protozoa in the Lower St. Lawrence Estuary: comparison with bacterial, phytoplankton, and particulate organic carbon. Aquat Microb Ecol 9:243-258

Smith BN, Epstein S (1971) Two categories of ${ }^{13} \mathrm{C} /{ }^{12} \mathrm{C}$ ratios for higher plants. Plant Physiol 47:380-384

Smith RJ, Hobson KA, Koopman HN, Lavigne DM (1996) Distinguishing between populations of fresh- and saltwater harbour seals (Phoca vitulina) using stable-isotope ratios and fatty acid profiles. Can J Fish Aquat Sci 53: 272-279

Sprules WG, Bowerman JE (1988) Omnivory and food chain length in zooplankton food webs. Ecology 69:418-426

Surif MB, Raven JA (1990) Photosynthetic gas exchange under emersed conditions in eulittoral and normally submersed members of the Fucales and Laminariales: interpretation in relation to $\mathrm{C}$ isotope and $\mathrm{N}$ and water use efficiency. Oecologia 82:68-80

Tan FC, Strain PM (1979) Organic carbon isotope ratios in recent sediments in the St Lawrence estuary and the Gulf of St Lawrence. Estuar Coast Shelf Sci 8:213-225

Tan FC, Strain PM (1983) Sources, sinks and distribution of organic carbon in the St. Lawrence Estuary, Canada. Geochim Cosmochim Acta 47:125-132

Therriault JC (1991) The Gulf of St. Lawrence: small ocean or big estuary? Can Spec Publ Fish Aquat Sci 113:1-359

Thompson D, Hammond PS, Nicholas KS, Fedak MA (1991) Movements, diving and foraging behaviour of grey seals (Halichoerus grypus). J Zool (Lond) 224:223-232

Thompson PM (1993) Harbour seal movement patterns. Symp Zool Soc (Lond) 66:225-239

Tieszen LL, Boutton TW, Tesdahl KG, Slade NA (1983) Fractionation and turnover of stable carbon isotopes in animal tissues: implications for $\delta^{13} \mathrm{C}$ analysis of diet. Oecologia 57: 32-37

Vladykov V (1946) Etudes sur les mammifères aquatiques. IV. Nourriture du marsouin blanc ou béluga (Delphinapterus leucas) du fleuve Saint-Laurent. Département des Pêcheries, Québec, QC

Werner EE, Gilliam JF (1984) The ontogenetic niche and species interactions in size-structured populations. Annu Rev Ecol Syst 15:393-425

Submitted: March 17, 2000; Accepted: August 24, 2000

Proofs received from author(s): December 13, 2000 